\title{
THERAPEUTIC EFFICACY OF INTRA-ARTICULAR DELIVERY OF ENCAPSULATED HUMAN MESENCHYMAL STEM CELLS ON EARLY STAGE OSTEOARTHRITIS
}

\author{
J.M. McKinney ${ }^{1,2,4}$, T.N. Doan ${ }^{1,2}$, L. Wang 3 , J.N. Deppen ${ }^{3,4}$, D.S. Reece, ${ }^{4,5}$, K.A. Pucha ${ }^{2}$, S.C. Ginn ${ }^{3}$, \\ R.D. Levit ${ }^{3}$ and N.J. Willett ${ }^{1,2,4,5, *}$ \\ ${ }^{1}$ Research Service, VA Medical Center, Decatur, GA, USA \\ ${ }^{2}$ Department of Orthopedics, Emory University, Atlanta, GA, USA \\ ${ }^{3}$ Department of Medicine, Division of Cardiology, Emory University, Atlanta, GA, USA \\ ${ }^{4}$ Wallace H. Coulter Department of Biomedical Engineering, Georgia Institute of Technology \\ and Emory University, Atlanta, GA, USA \\ ${ }^{5}$ Parker H. Petit Institute for Bioengineering and Bioscience, Georgia Institute of Technology, \\ Atlanta, GA, USA
}

\begin{abstract}
Mesenchymal stem cells (MSCs) represent a great therapeutic promise in pre-clinical models of osteoarthritis (OA), but many questions remain as to their therapeutic mechanism of action: engraftment versus paracrine action. Encapsulation of human MSCs (hMSCs) in sodium alginate microspheres allowed for the paracrine signaling properties of these cells to be isolated and studied independently of direct cellular engraftment. The objective of the present study was to quantitatively assess the efficacy of encapsulated hMSCs as a disease-modifying therapeutic for OA, using a medial meniscal tear (MMT) rat model. It was hypothesized that encapsulated hMSCs would have a therapeutic effect, through paracrine-mediated action, on early OA development.

Lewis rats underwent MMT surgery to induce OA. $1 \mathrm{~d}$ post-surgery, rats received intra-articular injections of encapsulated hMSCs or controls (i.e., saline, empty capsules, non-encapsulated hMSCs). Microstructural changes in the knee joint were quantified using equilibrium partitioning of a ionic contrast agent based micro-computed tomography (EPIC- $\mu \mathrm{CT}$ ) at 3 weeks post-surgery, an established time point for early OA.

Encapsulated hMSCs significantly attenuated MMT-induced increases in articular cartilage swelling and surface roughness and augmented cartilaginous and mineralized osteophyte volumes.

Cellular encapsulation allowed to isolate the hMSC paracrine signaling effects and demonstrated that hMSC s could exert a chondroprotective therapeutic role on early stage OA through paracrine signaling alone. In addition to this chondroprotective role, encapsulated hMSCs augmented the compensatory increases in osteophyte formation. The latter should be taken into strong consideration as many clinical trials using MSCs for OA are currently ongoing.
\end{abstract}

Keywords: Osteoarthritis, medial meniscal transection, human mesenchymal stem cells, cellular encapsulation, osteophytes, paracrine signaling, contrast enhanced micro-computed tomography.

*Address for correspondence: Nick J. Willett, Atlanta Veteran Affairs Medical Center, 1670 Clairmont Rd, Room 5A-115, Decatur, GA 30033, USA.

Telephone number: +1 4043216111 Email: nick.willett@emory.edu

Copyright policy: This article is distributed in accordance with Creative Commons Attribution Licence (http://creativecommons.org/licenses/by-sa/4.0/).

\section{Introduction}

Osteoarthritis (OA) is the most common joints' chronic disease and remains one of the leading global causes of disability (Cisternas et al., 2016; Vos et al., 2012). Disease incidence is expected to continue rising with the increasingly obese and aging global population (Vos et al., 2012). OA is characterized by chronic degeneration of articular cartilage, with proteoglycan loss, chondrocyte hypertrophy, matrix fibrillation, erosion and lesion formation and eventually fullthickness loss of articular cartilage resulting in 
bone-on-bone contact (Bertrand et al., 2010; MartelPelletier, 2004). OA-associated phenotypic changes are not strictly limited to articular cartilage, as surrounding tissue is additionally altered, with osteophyte formation, synovial inflammation and subchondral bone remodeling (Loeser et al., 2012). While physical therapy and weight loss improve functionality in patients with OA, current drugs (analgesics, viscosupplements, corticosteroid injections) only provide symptomatic relief (Messier et al., 2004; Yu and Hunter, 2016). There is a notable need for development of a disease-modifying therapeutic for OA. Mesenchymal stem cells (MSCs) represent a promising treatment to target the disease, relying on their regenerative capacity along with their immunomodulatory and anti-inflammatory properties.

MSC therapeutics for cartilage regeneration are widely studied, in both the pre-clinical and clinical environment. Pre-clinical studies demonstrate the efficacy of autologous, allogeneic and xenogeneic MSC intra-articular injections for cartilage regeneration (Hatsushika et al., 2013; Kim et al., 2014; Pigott et al., 2013; Saulnier et al., 2016). While varied results are reported in OA as to the efficacy of xenogeneic cell sources, this remains an understudied area (Farrell et al., 2016; Saulnier et al., 2016). However, recent in vivo data, following intra-articular injections, demonstrate that xenogeneic MSCs do not elicit an increased immune response, suggesting these cells may be immunoprivileged in the short-term (Pigott et al., 2013; Saulnier et al., 2016). While these pre-clinical studies show improved cartilage repair with MSC treatment, much remains unknown and MSCs have yet to be translated into an effective clinical therapy (Mamidi et al., 2016; Pers et al., 2015; Yu and Hunter, 2016; Web ref. 1). MSCs may be acting through direct engraftment, in addition to paracrine signaling, in concert with the local environment (Grigolo et al., 2009; Horie et al., 2012; Sato et al., 2012). However, MSC viability studies, following intra-articular injection, show short-term survival $(\sim 7 \mathrm{~d})$ and small numbers of engrafted cells, indicating that the newly regenerated tissue derives mostly from the host cells (Horie et al., 2012; Sato et al., 2012; ter Huurne et al., 2012). These findings suggest that the recruitment of endogenous cells may be a critical component to MSC therapies, through paracrine communication. Human MSCs (hMSCs) can secrete a wide range of paracrine factors to facilitate tissue remodeling, recruit stem and progenitor cells and modulate the immune response. In response to cartilage degeneration, hMSCs can induce tissue remodeling through secretion of factors such as interleukin (IL)- 6 and stromal-cell-derived factor (SDF)-1 (Liu et al., 2011; Namba et al., 2007). hMSCs can also modulate the host immune response factors tumor necrosis factor alpha (TNF- $\alpha$ )-stimulated gene (TSG)-6, transforming growth factor (TGF)- $\beta 1$ and adenosine (Choi et al., 2011; Kintscher et al., 2002; Kunzmann et al., 2006;
Shin et al., 2018). Additionally, in graft-versus-host disease, MSC apoptosis, following delivery in vivo by systemic administration, further modulates the immune response; however, it is unknown if this is important in the context of local treatment of osteoarthritic joints (Galleu et al., 2017). The relative contribution of hMSC paracrine factors, independent of direct cellular engraftment, is difficult to define.

Cellular encapsulation represents a promising means to isolate and study hMSC paracrine factors, independent of cellular engraftment, as the capsule provides a mechanical barrier between the encapsulated cells and the native host tissue. Sodium alginate, an inert heteropolysaccharide, is used as a vehicle to encapsulate various cell types for delivery (Orive et al., 2003). Sodium alginate capsules can be formed with controlled porosity, allowing encapsulated MSCs to communicate with the surrounding native tissue through paracrine mechanisms, while retaining the cells inside the microcapsules (Landázuri et al., 2012). Encapsulation of MSCs in semi-permeable alginate allows for diffusion of molecules into and out of the cell-containing microspheres, enabling them to sense signals from the diseased tissue and to secrete various factors into the surrounding environment. The molecular cut-off of proteins readily diffusible through the alginate capsule is $\sim 80 \mathrm{kDa}$ - through $24 \mathrm{~h}$ incubation of encapsulated fluorescent microspheres - thus allowing free transit of small cytokines, chemokines and growth factors while excluding larger proteins, such as IgG (Landázuri et al., 2012). In a murine hind limb ischemia model, encapsulation of xenogeneic hMSCs increases cell survival, retention and pro-angiogenic activity at the ischemic injury site and intact capsules are recoverable up to 5 months post-implantation (Landázuri et al., 2012). Furthermore, delivery of encapsulated xenogeneic hMSCs in a hydrogel patch post rat myocardial infarction demonstrates increased viable cell retention at the delivery site, improved cardiac function, decreased scar size and increased microvascular density (Levit et al., 2013). The improved retention of hMSCs using this delivery method could be explained by alginate's partial protection from the host's immune-mediated clearance. Encapsulation of hMSCs was used in the present study to isolate the hMSC paracrine signaling properties from direct engraftment and to study the associated effects on the onset of OA.

There are currently many ongoing clinical trials using MSCs, but these have yet to translate into an effective clinical therapy (Mamidi et al., 2016; Pers et al., 2015; Yu and Hunter, 2016; Web ref. 1). In contrast, MSCs repeatedly show great promise in pre-clinical studies (Mamidi et al., 2016; Pers et al., 2015; Yu and Hunter, 2016). This motivates more detailed investigations into the MSC mechanism of action. Particularly, the role of paracrine action versus direct cellular engraftment of these cells need to be explored 
further to better design cell-based therapies that will effectively translate to the human scale. The objective of the study was to examine the therapeutic efficacy of hMSCs with and without encapsulation in early stage post-traumatic OA. Equilibrium partitioning of a ionic contrast agent based micro-computed tomography (EPIC- $\mu \mathrm{CT}$ ) was used to quantitatively analyze changes in articular cartilage, osteophyte and subchondral bone morphologies. EPIC- $\mu \mathrm{CT}$ is established and validated in reference to the gold standard in the musculoskeletal field, histopathology (Palmer et al., 2006; Reece et al., 2017; Thote et al., 2013; Willett et al., 2014; Willett et al., 2016; Xie et al., 2010). The hypothesis was that encapsulated hMSCs would have a therapeutic effect, through paracrine-mediated action, on the onset and development of early stage OA.

\section{Materials and Methods}

\section{Surgical methods}

Animal care and experiments were conducted in accordance with the institutional guidelines of the Atlanta Veteran Affairs Medical Center (VAMC) and experimental procedures were approved by the Atlanta VAMC Institutional Animal Care and Use Committee (IACUC) (Protocol: V004-15).

Weight-matched wild type male Lewis rats (strain code: 004; Charles River), weighing 300-350 g, were acclimatized for 1 week after they were received. A surgical instability animal model, medial meniscal tear (MMT), was used to induce OA (Bendele, 2001). Animals were anesthetized using isoflurane and injected subcutaneously with $1 \mathrm{mg} / \mathrm{kg}$ sustainedrelease buprenorphine (ZooPharm, Windsor, CO, USA). Skin over the medial aspect of the left femoro-tibial joint was shaved and sterilized. Blunt dissection was used to expose the medial collateral ligament (MCL), which was next transected to expose the meniscus. Then, a full-thickness cut was made through the meniscus at its narrowest point. Following transection of the meniscus, soft tissues were re-approximated and closed using 4.0 Vicryl sutures and the skin was closed using wound clips. Sham surgery was also performed in rats. For shams, the MCL was transected followed by closure of the skin without transection of the meniscus.

$1 \mathrm{~d}$ post-surgery, MMT animals received $50 \mu \mathrm{L}$ intra-articular injections using a 25-gauge needle. Animals were injected with i) Hanks balanced salt solution (HBSS) $(n=8)$, ii) empty sodium alginate capsules $(n=7)$, iii) $5 \times 10^{5} \mathrm{hMSC} /$ knee in HBSS $(n=8)$ or iv) $5 \times 10^{5}$ encapsulated hMSC (encap hMSC)/ knee $(n=8)$. The cell dose $\left(5 \times 10^{5}\right.$ cells $/$ knee $)$ used for injection was the maximum concentration that could be encapsulated and delivered in a $50 \mu \mathrm{L}$ volume. Sham $(n=8)$ animals were not injected post-surgery.

3 weeks post-surgery, animals were euthanized by $\mathrm{CO}_{2}$ inhalation. The MMT surgical model shows initial phenotypical changes associated with OA at the standard 3 weeks post-surgery time point, including cartilage damage, osteophyte formation and subchondral bone changes (Bendele, 2001). This time point was matched with animal euthanasia to assess the therapeutic effects of encapsulated hMSCs on the onset and early development of OA. Left hind limbs were collected and fixed in $10 \%$ neutral buffered formalin.

\section{Cell culture and characterization}

hMSCs derived from bone marrow were obtained from Emory Personalized Cell Therapy core facility at Emory University. hMSCs were cultured in complete minimum essential medium Eagle- $\alpha$ modification ( $\alpha$-MEM; 12561; Gibco) supplemented with $10 \%$ heat-inactivated fetal bovine serum (FBS) $(\mathrm{S} 11110 \mathrm{H}$; Atlanta Biologicals, Lawrenceville, GA, USA), 2 mM L-glutamine (SH3003401; HyClone, Logan, UT, USA) and $100 \mu \mathrm{g} / \mathrm{mL}$ penicillin/streptomycin (P/S) (B21110; Atlanta Biologicals) and sub-cultured at $70 \%$ confluency. For lentiviral preparation, HEK 293T cells (CRL-3216TM; ATCC) were cultured in complete Dulbecco's modified Eagle's medium (DMEM; D5546; Sigma-Aldrich). hMSC phenotype was confirmed by adipogenic, chondrogenic and osteogenic differentiation (kit protocols A1007001, A1007101, A1007201; StemPro ${ }^{\mathrm{TM}}$ Differentiation Kits, ThermoFisher Scientific). Flow cytometry was also used to characterize the hMSCs. A hMSC Verification Flow Kit (FMC020; R\&D Systems) was used to confirm that MSCs expressed characteristic MSC surface markers (CD73, CD90, CD105) and lacked hematopoietic markers (CD45, CD34, CD11b, CD79A, HLA-DR).

\section{Encapsulation of hMSCs}

$1 \times 10^{6}$ cells/mL passage $4 \mathrm{hMSC}$ sere suspended in $1 \%$ ultrapure low viscosity sodium alginate LVG (UP-LVG) (4200006; PRONOVA ${ }^{\mathrm{TM}}$ UP LVG; NovaMatrix, Sandvika, Norway). An electrostatic encapsulator (VARV1; Nisco Engineering AG, Zurich, Switzerland) with a $0.2 \mu \mathrm{m}$ nozzle, $2.5 \mathrm{~mL} / \mathrm{h}$ flow rate and $7 \mathrm{kV}$ voltage was used. Capsules were gelled in $50 \mathrm{mM} \mathrm{BaCl}$. hMSC capsules were washed six times with $0.9 \%$ saline $(\mathrm{NaCl})$, re-suspended to the appropriate dose and stored at $4{ }^{\circ} \mathrm{C}$ in saline until injection. hMSC viability was confirmed with Live/ Dead $^{\mathrm{TM}}$ Viability/Cytotoxicity kit (L3224; Invitrogen) following encapsulation. Cell viability was quantified using ImageJ software. All rats were injected within $2 \mathrm{~h}$ of hMSC encapsulation.

\section{In vitro capsule viability study}

Immediately following encapsulation and washing, encapsulated hMSCs were placed in complete $\alpha$-MEM medium in 12-well plates and cultured at $37^{\circ} \mathrm{C}, 5 \%$ $\mathrm{CO}_{2}$ until different time points were reached $(1,3,5$, $7,9,14,21,28$ and 35 d post plating), with medium changed every $3 \mathrm{~d}$. At the specified time point, hMSC viability in capsules was determined with Live/Dead ${ }^{\mathrm{TM}}$ Viability/Cytotoxicity kit (Invitrogen). 
Percentage viability was quantified by comparing the relative number of live cells stained with calcein-AM to ethidium-homodimer-stained dead cells counted through ImageJ software on serial z-stacked images, each containing 3-17 capsules, obtained by a confocal microscope, at $3.99 \mu \mathrm{m} z$-thickness. Image collection and quantification was completed for every 14 sections, ensuring encapsulated cells were counted only once.

\section{Lentiviral vector packaging}

The lentiviral vector, pLenti CMV Puro LUC (w168-1) (\#17477; Addgene, Cambridge, MA, USA), contains a CMV promoter upstream of the firefly luciferase gene and a murine phosphoglycerate kinase (pGK) promoter upstream of a puromycin resistance gene (Campeau et al., 2009). Second generation packaging plasmids were used for lentiviral packaging. pLenti CMV Puro LUC was co-transfected with a packaging plasmid, psPAX2 (\#12260; Addgene) and a VSVG-envelope-expressing plasmid, pMD2.G (\#12259; Addgene), in a $4: 2: 1$ ratio into HEK 293T cells using Lipofectamine ${ }^{\mathrm{TM}} 3000$ Transfection Reagent (L3000008; Invitrogen) and Opti-MEM ${ }^{\mathrm{TM}}$ I Reduced Serum Medium (31985062; ThermoFisher Scientific), as recommended by the manufacturer. $70 \%$ confluent HEK 293T cells were transfected with the plasmid mixture in transfection medium (DMEM + $10 \%$ FBS + 2 mM glutamine). Transfection medium was replaced with collection medium (DMEM + $10 \%$ $\mathrm{FBS}+2 \mathrm{mM}$ glutamine $+100 \mu \mathrm{g} / \mathrm{mL} \mathrm{P} / \mathrm{S}$ at $18 \mathrm{~h}$ posttransfection. Collection medium was harvested at 41, 48 and $65 \mathrm{~h}$ post-transfection, pooled, centrifuged at $400 \times g$ for $5 \mathrm{~min}$ at $4{ }^{\circ} \mathrm{C}$ and filtered through a $0.45 \mu \mathrm{m}$ polyethersulfone membrane syringe filter (6780-2504; Whatman). The viral supernatant was concentrated using the PEG-it Virus Precipitation Solution (LV810A; System Biosciences, Palo Alto, CA, USA) and stored at $-80^{\circ} \mathrm{C}$.

\section{hMSC lentiviral transduction}

50-60 \% confluency passage $3 \mathrm{hMSC}$ s were transduced with $7 \mathrm{~mL}$ of concentrated viral supernatant in T175 flasks (353112; Corning) with $8 \mu \mathrm{g} / \mathrm{mL}$ polybrene (AL118; Sigma-Aldrich) in complete DMEM containing $10 \%$ FBS. Antibiotic selection commenced $48 \mathrm{~h}$ posttransduction and continued throughout culture by replacing medium with DMEM supplemented with $10 \%$ FBS, 2 mM glutamine, $100 \mu \mathrm{g} / \mathrm{mL}$ P/S and $0.6 \mu \mathrm{g} /$ mL puromycin (H9268; Sigma-Aldrich). Transduced hMSCs were passaged when reaching 60-90\% confluency. Luciferase expression was confirmed through addition of D-luciferin (L-123-250; Gold Biotechnology, St. Louis, MO, USA) to transduced hMSCs, with luminescence measurements made with a microplate luminometer (BioTek SynergyTM H1, Winooski, VT, USA). The hMSCs transduced with the lentivirus were used for bioluminescent-cell-tracking studies only. The hMSCs injected for therapeutic efficacy studies evaluated by EPIC- $\mu \mathrm{CT}$ analysis did not contain the firefly luciferase gene.

\section{Bioluminescence imaging (BLI)}

Naïve Lewis rats were injected intra-articularly with $5 \times 10^{5}$ cells/knee of either encapsulated or nonencapsulated luciferase-expressing hMSCs $(n=5$ for each condition). Following cellular injections at day 0 , animals received an intra-articular injection of $40 \mathrm{mg} / \mathrm{mL}$ luciferin (Promega ${ }^{\mathrm{TM}}$ Beetle Luciferin, Potassium Salts, ThermoFisher Scientific) diluted in $\alpha$-MEM (12561; Gibco). Incubation times for initial and subsequent luciferin injections were optimized in a prior pilot study, in which incubation time points that yielded maximum signal were selected, using the Bruker In-Vivo Xtreme. At day 0, a 30 min incubation time was allotted before BLI was conducted using the Bruker In-Vivo Xtreme imaging system. Additional BLI readings were performed at 1, 3, 5, 7 and $9 \mathrm{~d}$ post hMSC injections, with subsequent luciferin injections administered $20 \mathrm{~min}$ (incubation time) before readings. The minimum detection limit for luciferaseexpressing hMSCs, in vitro, using the In-Vivo Xtreme imaging system was determined to be 10,000 cells (data not shown). Bioluminescence intensity values were quantified using ImageJ software and plotted as percentage of maximum intensity. Background (naïve animals with luciferin alone) images $(n=4)$ were also collected and the averaged intensity value was subtracted from intensity values collected for all study samples.

\section{EPIC- $\mu C T$ analysis of articular cartilage, osteophytes and subchondral cone}

EPIC- $\mu C T$ was used to quantitatively evaluate articular cartilage structure and composition, osteophyte volumes and subchondral bone morphology (Fig. 1), as previously described (Palmer et al., 2006; Reece et al., 2017; Thote et al., 2013; Willett et al., 2014; Willett et al., 2016; Xie et al., 2010). Fixed tibiae were dissected, carefully exposing the articular cartilage of the medial tibial plateau and stored in phosphate-buffered saline (PBS). Before scanning, the proximal tibia was immersed for $30 \mathrm{~min}$ at $37^{\circ} \mathrm{C}$ in $2 \mathrm{~mL}$ of $30 \%$ Hexabrix ${ }^{\circledR} 320$ contrast reagent (NDC 67684-5505-5; Guerbet, Villepinte, France) and $70 \%$ PBS (Palmer et al., 2006). Tibiae were gently patted dry, placed in covered sample chambers and scanned using Scanco MCT 40 (Scanco Medical, Brüttisellen, Switzerland). Scan parameters were: $45 \mathrm{kVp}, 177 \mu \mathrm{A}$, $200 \mathrm{~ms}$ integration time, $16 \mu \mathrm{m}$ voxel size, $~ 27$ min scan time (Palmer et al., 2006). Raw scan data were automatically reconstructed to two-dimensional (2D) grayscale tomograms and orthogonally transposed to yield coronal sections.

Coronal sections were evaluated in the medial $1 / 3$ of the medial tibial plateau for articular cartilage parameters, as this region demonstrates high damage incidence in the MMT-induced OA model (Thote et al., 2013). Contours were constructed around medial tibial cartilaginous surfaces. Thresholding was used to separate cartilage from air and bone, on the medial $1 / 3$, with thresholds of 110 and $435 \mathrm{mg}$ hydroxyapatite per cubic centimeter $\left(\mathrm{mg} \mathrm{HA} / \mathrm{cm}^{3}\right)$, respectively (Fig. 
$1 \mathbf{b}, \mathbf{c})$. Results yielded average articular cartilage volume, thickness and attenuation. Cartilage attenuation is a parameter inversely proportional to sulfated glycosaminoglycan (sGAG) content (Palmer et al., 2006). More specifically, degraded cartilage has a lower sGAG content so that a larger amount of contrast agent integrates into the cartilage matrix and, therefore, reflects a higher attenuation value (Palmer et al., 2006).

Osteophytes are defined as a thickening of tissue on the most medial aspect of the tibial condyle (van der Kraan and van der Berg, 2007). Osteophyte volume is evaluated using the coronal section (Reece et al., 2017; Willett et al., 2016). The osteophyte cartilage volume was measured in volumes of interest that excluded the peripheral soft tissue. For cartilaginous osteophyte volumes, threshold values of 110-435 mg $\mathrm{HA} / \mathrm{cm}^{3}$ were used to segment the cartilage from air and bone. For mineralized osteophyte volume measurements, thresholds of $435-1000 \mathrm{mg} \mathrm{HA} / \mathrm{cm}^{3}$ were used to exclude mineralized tissue volumes from surrounding cartilage, soft tissue and air (Fig. 1f-i). Total osteophyte volumes were calculated by adding the respective volumes of cartilaginous and mineralized osteophytes.

Subchondral bone was analyzed over the entire medial tibial plateau using coronal sections. Thresholds of $435-1000 \mathrm{mg} \mathrm{HA} / \mathrm{cm}^{3}$ were used to separate the bone from the surrounding cartilage and soft tissue (Fig. 1d,e). Average subchondral bone volume, thickness, attenuation (density) and porosity were measured.

\section{Surface roughness analysis of articular cartilage} Serial coronal sections of the medial tibial plateau, obtained from EPIC- $\mu \mathrm{CT}$ scans, were exported as 2D grayscale TIFF images and imported into MATLAB R2017a (MathWorks, Natick, MA, USA). A custom algorithm scanned section images sequentially to create three-dimensional (3D) digital representations

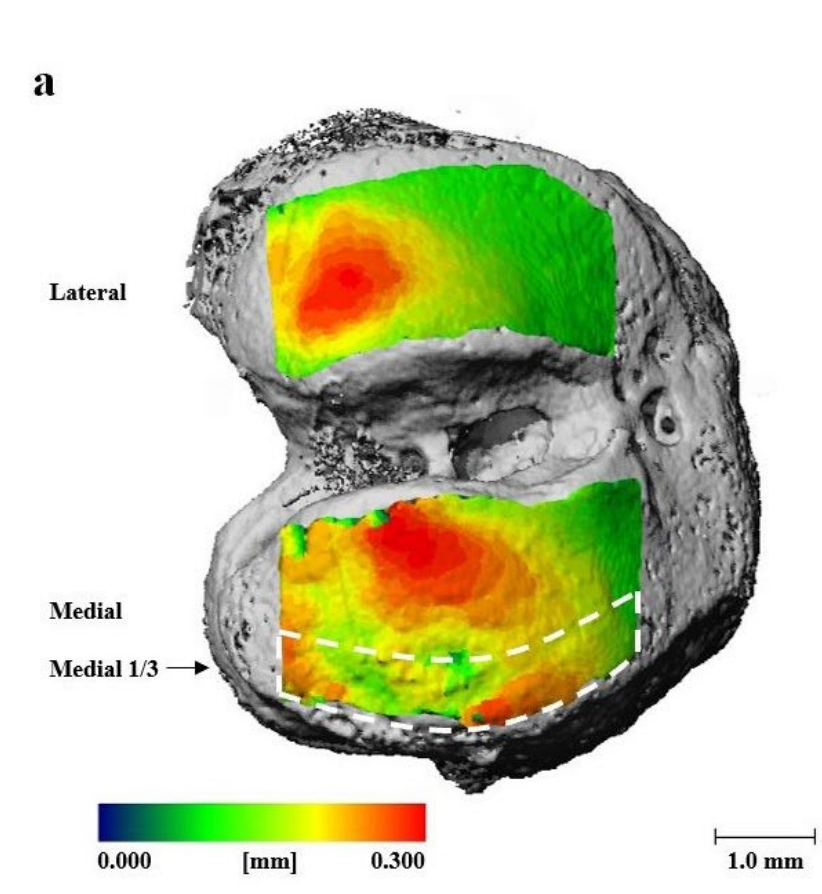

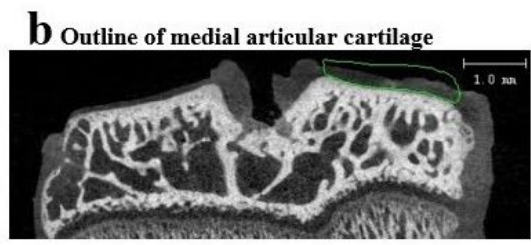

C Medial 1/3 of medial articular cartilage
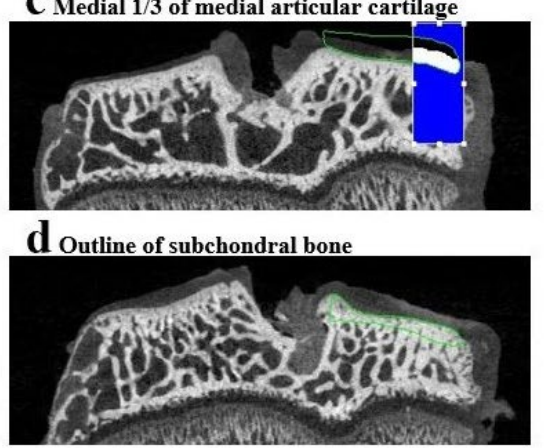

e Total medial subchondral bone

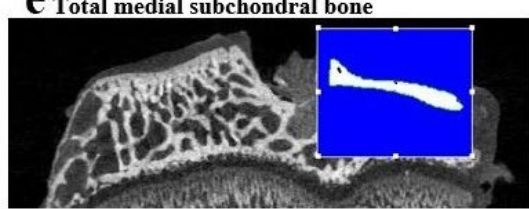

h Cartilaginous osteophyte
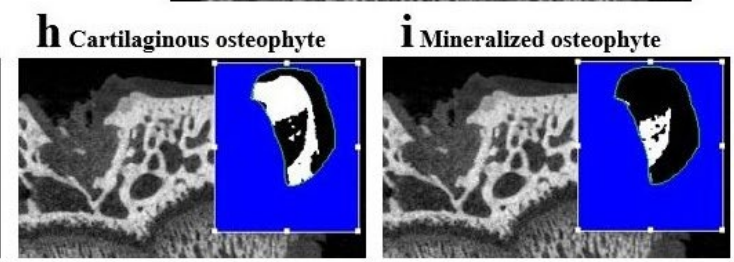
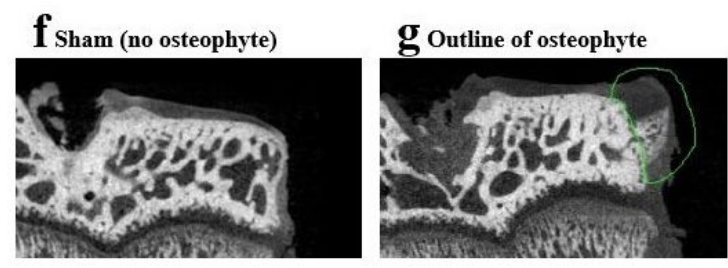

Fig. 1. Representative EPIC- $\mu \mathrm{CT}$ images demonstrating articular cartilage, osteophyte and subchondral bone volume of interest (VOI). (a) Rat tibial articular cartilage thickness map overlays on bone indicating articular cartilage VOI. Color heat map indicates cartilage thickness according to the scale bar (blue $\sim 0.0 \mathrm{~mm}$; red $\sim 0.3 \mathrm{~mm}$ ) (b) Representative images of coronal sections of rat tibial joint showing outline of total medial articular cartilage, (c) medial $1 / 3$ (indicated in white) of medial articular cartilage, outline of (d) medial tibial subchondral bone and (e) cortical subchondral bone (indicated in white) of medial tibia. Representative coronal sections of medial tibial joint illustrating (f) no osteophyte in sham joint, (g) contour of osteophyte in MMT joint, (h) cartilaginous osteophyte volume (indicated in white) in MMT joint and (i) mineralized osteophyte volume (indicated in white) in MMT joint. Scale bar in (a) is universal for all representative images of coronal sections. 
of respective cartilaginous surfaces (Reece et al., 2017). Briefly, cartilage surfaces were fitted with a 3D polynomial surface: fourth order along the ventraldorsal axis and second order along the medial-lateral axis. Surface roughness was calculated as the root mean square of differences between the articular cartilage surface and the polynomial fitted surface. Surface roughness was analyzed over the medial $1 / 3$ of the medial tibial plateau.

\section{Histology}

Tibiae were decalcified with Immunocal (SKU1414-32; StatLab, McKinney, TX, USA) for 7-10 d. Dehydrated samples were processed into paraffinembedded blocks, $5 \mu \mathrm{m}$-thick sectioned and stained with hematoxylin and eosin (H\&E; Fisherbrand ${ }^{\mathrm{TM}}$ 517-28-2, Waltham, MA, USA) or safranin O and fast green (Saf-O; Electron Microscopy Sciences ${ }^{\circledR}$ 20800, Hatfield, PA, USA), following manufacturer protocols.

\section{Statistical analysis}

All quantitative data are presented as mean \pm standard deviation (SD). The BLI study was evaluated by repeated measures using two-way analysis of variance (ANOVA) (factors: time point and delivery method) with post-hoc Tukey Honest analysis. Articular cartilage and subchondral bone parameters were evaluated using one-way ANOVA with post-hoc Tukey Honest analysis. Osteophyte parameters were evaluated using one-way ANOVA with a nonparametric post-hoc Bonferroni analysis. Statistical significance was set at $p<0.05$. All data were analyzed using GraphPad Prism software version 6.0 (GraphPad Software Inc., La Jolla, CA, USA).

\section{Results}

\section{Encapsulated hMSC characterization}

To characterize the hMSCs used for therapeutic delivery, differentiation and phenotypic characterization assays were performed prior to encapsulation. hMSC differentiation was confirmed with immunofluorescence staining for type II collagen of paraffin-sectioned pellets, oil red $\mathrm{O}$ staining and alizarin red $S$ staining for chondrogenesis, adipogenesis and osteogenesis, respectively (Fig. 2a-c). Additionally, hMSCs were confirmed to be positive for typical MSC markers, including CD73, CD90 and CD105, and negative for hematopoietic markers, including CD45, CD34, CD11b, CD79A and HLA-DR, by fluorescence-activated cell sorting analysis (FACS) (Fig. 2d).

hMSC were encapsulated in sodium alginate microspheres. The average diameter of encapsulated hMSC microspheres was $144 \pm 16 \mu \mathrm{m}$ (Fig. 2e). Encapsulated hMSC viability immediately following encapsulation was $96 \pm 2.4 \%$ (Fig. 2 f).

\section{Encapsulated hMSC tracking following intra- articular injection}

Cellular encapsulation improves MSC survival in both hindlimb ischemia and myocardial infarct models (Landázuri et al., 2012; Levit et al., 2013). However, no studies show the same augmented cell survival in knee joints following intra-articular delivery of encapsulated hMSCs. Luciferase-expressing hMSCs were used to analyze the effect of encapsulation on cellular viability, retention, proliferation and metabolic state both in culture and following intraarticular injections in rat knees (Allen et al., 2014; Levit et al., 2013). In vitro viability studies demonstrated that encapsulated hMSCs were approximately $75 \%$ viable for the first $7 \mathrm{~d}$ following encapsulation and remained approximately $30 \%$ viable for at least $35 \mathrm{~d}$ under standard culture conditions (Fig. 3a). Representative maximum projection images are included displaying encapsulated cells at four key study time points (Fig. 3b).

In vivo bioluminescence was plotted as percentage of maximum intensity, with maximum intensity (100\%) being expressed at day 0 for all animals. Encapsulated and non-encapsulated hMSCs showed similar initial decreases in bioluminescence, as no differences in signal were detectable for the first three study time points (day 0, 1 and 3). However, at later time points (day 5 and 7), non-encapsulated hMSCs had a small but statistically significant decrease in bioluminescence when compared to encapsulated hMSCs. While complete loss of hMSC bioluminescent signal ( $<1 \%$ of original intensity) was observed at day 7 , encapsulated hMSCs yielded only $\sim 6 \%$ cellular bioluminescence at this time point, with complete clearance at day 9 (Fig. 3c). Qualitative data, in the form of representative images selected from key time points for both groups, are included (Fig. 3d).

Full joint histology was performed on the hind limbs of animals ( $n=2 /$ time point) injected with encapsulated hMSCs, at 3 and 9 d post-injection, to qualitatively assess cell and capsule retention following intra-articular injection. Capsules can be readily visualized with $\mathrm{Saf}-\mathrm{O}$, a cationic stain that binds to negatively charged alginate (Schmitz et al. 2010). Intact alginate capsules containing hMSCs were visible within the infrapatellar fat pad of the knee at day 3 (Fig. 4a-c). Alginate capsules were also visible in the joint space at day 9, surrounded by capsule remnants (Fig. 4d,e). While no hMSCs could be identified within the capsules at day 9, lacunae empty or potentially containing cell debris were present in the capsules. Identification of encapsulated hMSCs at day 3 and the absence of hMSCs in alginate capsules at day 9 was consistent with the in vivo bioluminescent analysis (Fig. 3c).

\section{Qualitative analysis of the therapeutic efficacy of encapsulated hMSCs in OA}

Encapsulated hMSCs were injected $1 \mathrm{~d}$ post-surgery to assess the effects of the paracrine signaling 
properties of hMSCs, independent of direct cell engraftment, in delaying the onset of MMT-induced OA. Tibiae were collected 3 weeks post-surgery, the time point in the MMT model corresponding to the presentation of OA-associated phenotypical cartilage degeneration (Janusz et al., 2002). Histology was performed on collected tibiae to qualitatively analyze effects of encapsulated hMSC therapeutics on OA. Representative histological images of coronal tibial sections showed proteoglycan loss, degeneration of the cartilage surfaces and development of cartilaginous osteophytes in all MMT conditions (Fig. 5b-e,g-j). The sham group showed good proteoglycan staining and smooth cartilage surfaces with no osteophyte development (Fig. 5a,f). However, no further analysis was performed on $\mathrm{H} \& \mathrm{E}$ and Saf-O images as EPIC- $\mu$ CT was used to quantitatively analyze the therapeutic efficacy of encapsulated hMSCs on OA. Representative coronal slices from both histology and EPIC- $\mu$ CT showed qualitatively similar disease progression over the 3-week study time course (Fig. 5).

\section{EPIC- $\mu C T$ quantitative analysis of articular cartilage in the MMT study}

EPIC- $\mu C T$ was implemented to quantitatively analyze the effects of encapsulated hMSCs on MMTinduced OA development. Changes in articular cartilage structure and composition of the medial $1 / 3$ of the medial tibial condyle were quantified in 3D. Representative coronal cuts of contrast-enhanced cartilage EPIC- $\mu \mathrm{CT}$ attenuation maps of the whole medial tibial plateau showed qualitatively higher attenuation of cartilage for the MMT + saline (MMT/ saline), MMT + empty capsules (MMT/empty caps), MMT + hMSCs (MMT/hMSC) and MMT + encapsulated hMSCs (MMT/encap hMSCs) when compared to sham control (red = high attenuation, low sGAG content; green = low attenuation, high sGAG content) (Fig. 5k-o). Quantitative analysis of EPIC- $\mu$ CT images within the medial $1 / 3$ of the medial tibial plateau showed a significant increase in attenuation, representative of a decrease in sGAG content, for all MMT conditions as compared to the sham control (Fig. 6a). However, no significant

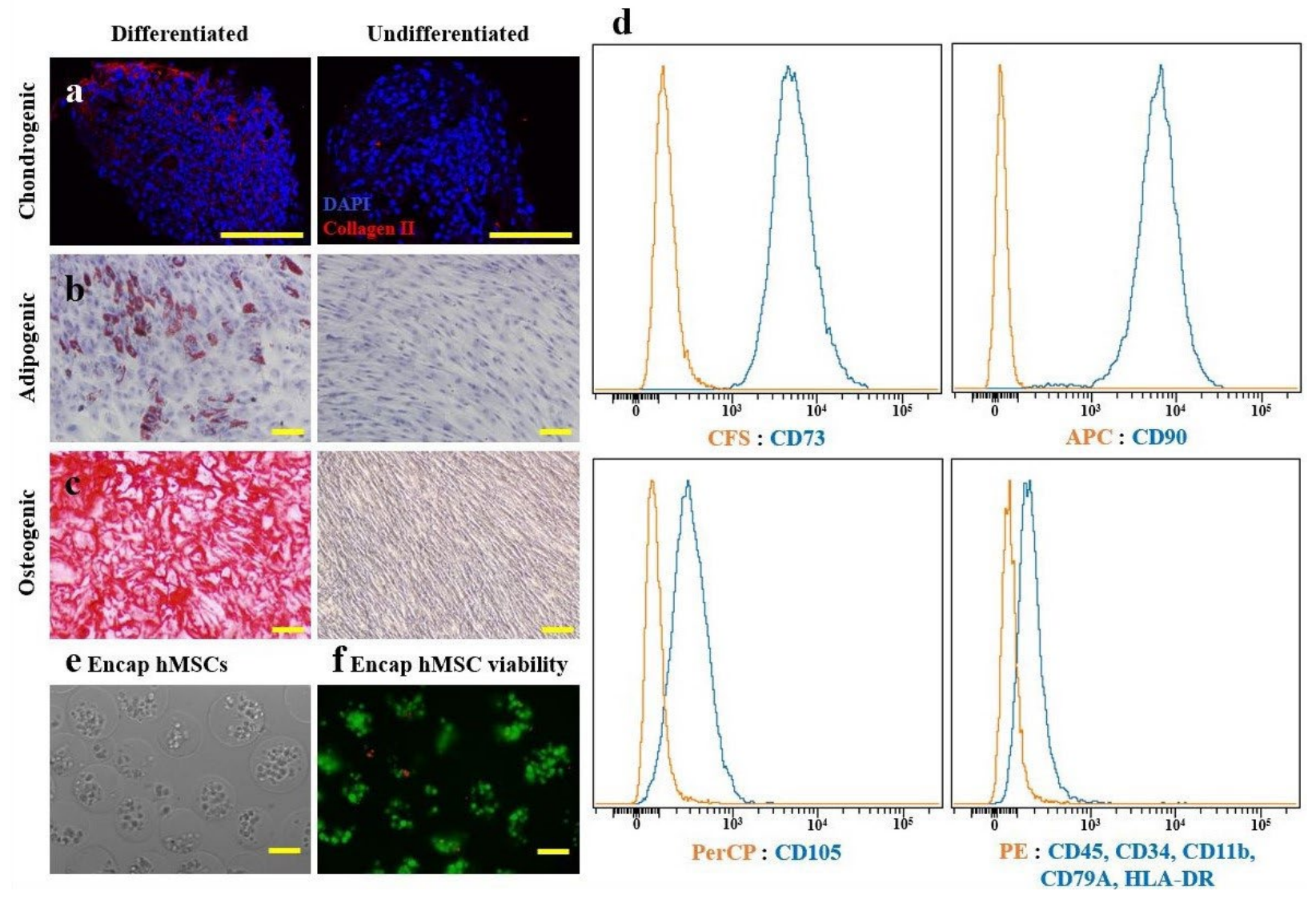

Fig. 2. Encapsulated hMSC characterization. Multipotency of hMSCs confirmed prior to encapsulation, as cultured hMSCs were differentiated into (a) chondrogenic, (b) adipogenic and (c) osteogenic phenotypes as demonstrated by collagen type II, oil red $\mathrm{O}$ and alizarin red staining. (d) FACS analysis demonstrated that hMSCs expressed typical MSC surface markers: CD73, CD90 and CD105; but not hematopoietic markers: CD45, CD34, CD11b, CD79A and HLA-DR. (e) Light microscopic appearance of encapsulated hMSCs immediately following sodium alginate encapsulation showed capsule diameters of $170 \pm 27 \mu \mathrm{m}$. (f) Fluorescent viability assay, staining live cells green and dead cells red, showed $96 \pm 2.4 \%$ of cells were viable immediately following encapsulation in sodium alginate. Scale bars $=100 \mu \mathrm{m}$. 
differences in attenuation were noted between any of the MMT conditions. Cartilage thickness of the medial $1 / 3$ of the medial tibial condyle showed significant increases in thickness values for all MMT conditions in comparison to the sham control (Fig. 6b). Additionally, the encapsulated hMSCs condition yielded attenuated cartilage thickness increases in comparison to all other MMT conditions. MATLAB surface roughness analysis of the medial $1 / 3$ of the medial tibial plateau showed an increased articular cartilage surface roughness for all MMT conditions as compared to sham animals (Fig. 6c). Furthermore, the encapsulated hMSC condition yielded attenuated surface roughness values in comparison to all other MMT conditions. Additionally, the saline group yielded augmented surface roughness in comparison to all MMT conditions. Qualitative analysis of the surface roughness was performed by subtracting individual $3 \mathrm{D}$ polynomial surfaces from the corresponding cartilage surface renderings, relative to the sham control (Fig. 6d-h).

EPIC- $\mu C T$ quantitative analysis of osteophytes and subchondral bone in MMT study

Osteophytes are a thickening and partial mineralization of cartilage tissue at the marginal edge of the medial tibial plateau and are a staple of OA development (van der Kraan and van der Berg, 2007). Cartilaginous and mineralized tissue volumes on the most medial aspect of the medial tibial condyle were quantified in 3D by EPIC- $\mu$ CT. Total osteophyte volume (cartilaginous + mineralized tissue volume) was significantly larger for all MMT conditions in comparison to the sham control (Fig. 7a). Furthermore, encapsulated hMSCs yielded significantly larger volumes than both the saline and empty capsule groups but not than the hMSC group. Cartilaginous osteophyte volumes were also significantly larger
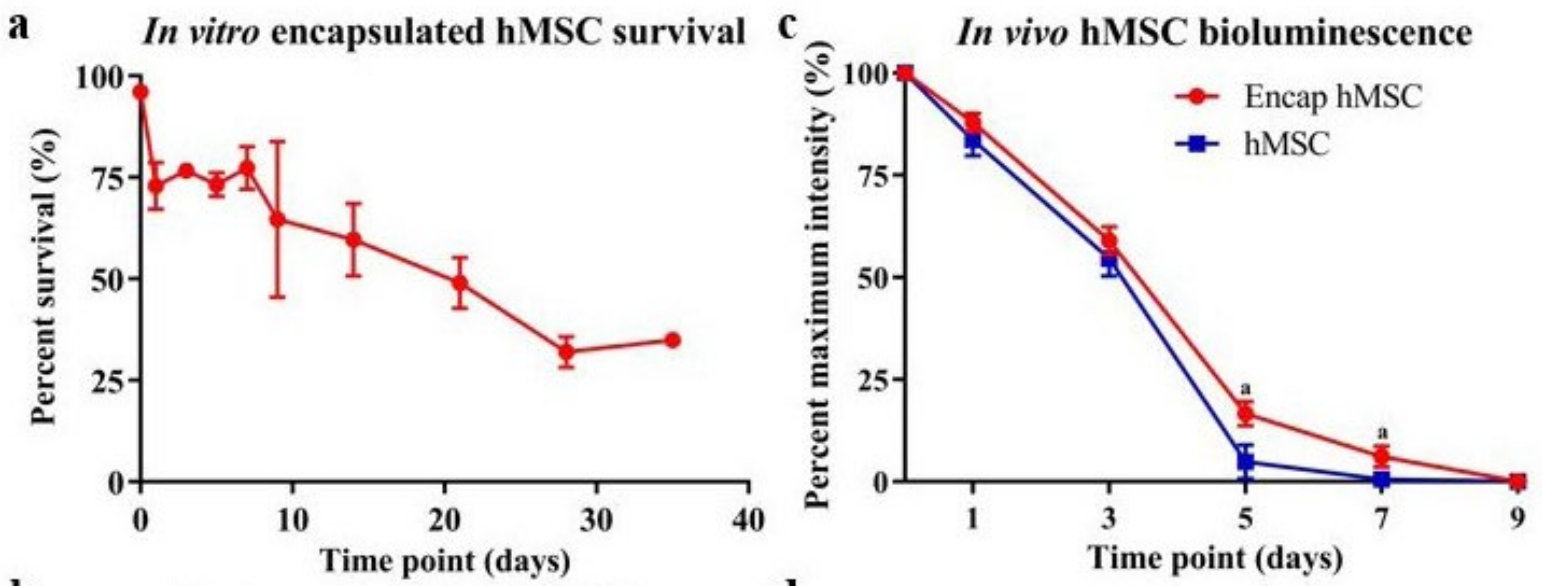
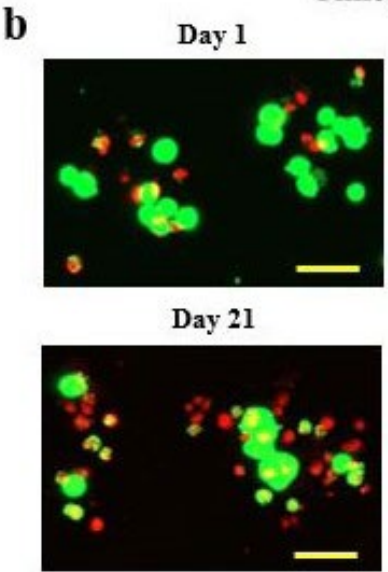

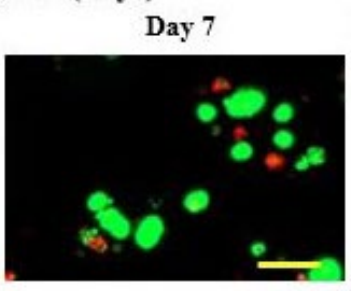

Day 35

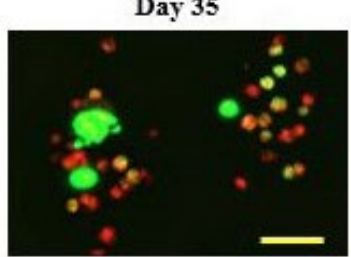

d

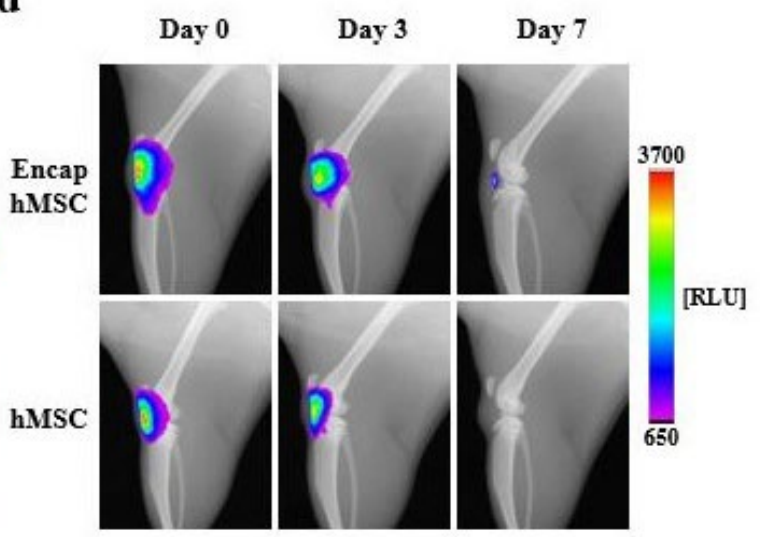

Fig. 3. Encapsulated hMSC in vitro viability and in vivo tracking following intra-articular injection into naïve joints. (a) hMSCs in alginate capsules remained $70-80 \%$ viable at early time points (days 1-7), with viability slowly declining to approximately $30 \%$ at day 28 and 35. (b) Representative maximum projection images of capsules from key time points qualitatively demonstrated encapsulated hMSC viability over time in vitro. (c) In vivo bioluminescent imaging demonstrated an overall increase in quantified bioluminescence of encapsulated hMSCs versus hMSCs. Initial time points showed similar bioluminescent signals, while later time points (day 5 and 7) demonstrated differences in bioluminescent signal. Complete clearance of hMSCs $(<1 \%)$ was observed at day 7 and complete clearance of encapsulated hMSCs was observed at day 9. (d) Representative images, from key study time points, of the rat knee joint qualitatively illustrate bioluminescent signal for each of the study groups. RLU: relative luminescence units with a color heat map according to the scale bar. Data presented as mean \pm SD. $n=5$ /group for in vivo study. ${ }^{a} p<0.05$. Scale bars $=50 \mu \mathrm{m}$. 
for all MMT conditions in comparison to the sham control (Fig. 7b). Furthermore, encapsulated hMSCs also showed increased cartilaginous osteophyte volumes in comparison to all other conditions, except hMSCs. Histological and EPIC- $\mu$ CT representative images qualitatively confirmed the results of the cartilaginous osteophyte volume analysis (Fig. $5 \mathbf{f}-\mathbf{j}, \mathbf{k}-\mathbf{o})$. Mineralized osteophyte volumes showed an increase for all MMT conditions as compared to the sham control (Fig. 7c). Additionally, encapsulated hMSCs yielded increased mineralized osteophyte volumes in comparison to all other experimental groups. EPIC- $\mu$ CT representative images qualitatively confirmed the results of the mineralized osteophyte volume analysis (Fig. 5k-o). Subchondral bone, the layer of bone just below the articular cartilage in load-bearing joints, showed an increased thickness in all MMT conditions as compared to the sham control (Fig. 7d). No significant differences were found between any of the MMT conditions for this parameter.

\section{Discussion}

The clinical need for disease-modifying drugs for the treatment of OA remains unmet. MSCs represent a promising treatment to target OA, relying on their regenerative capacity in combination with their immunomodulatory and anti-inflammatory properties (Choi et al., 2011; Kintscher et al., 2002; Kunzmann et al., 2006; Liu et al., 2011; Namba et al., 2007; Shin et al., 2018). Numerous clinical trials using MSCs are ongoing, but these have yet to translate into an effective clinical therapy, despite MSCs showing great promise on the pre-clinical side (Mamidi et al., 2016; Pers et al., 2015; Yu and Hunter, 2016; Web ref. $1)$. This motivates more detailed investigations into the MSC mechanism of action. More specifically, the main mechanism of action of these cells following intra-articular injection into the knee space: paracrine action versus cellular engraftment. The objective of the study was to quantitatively assess the efficacy of encapsulated hMSCs as a disease-modifying
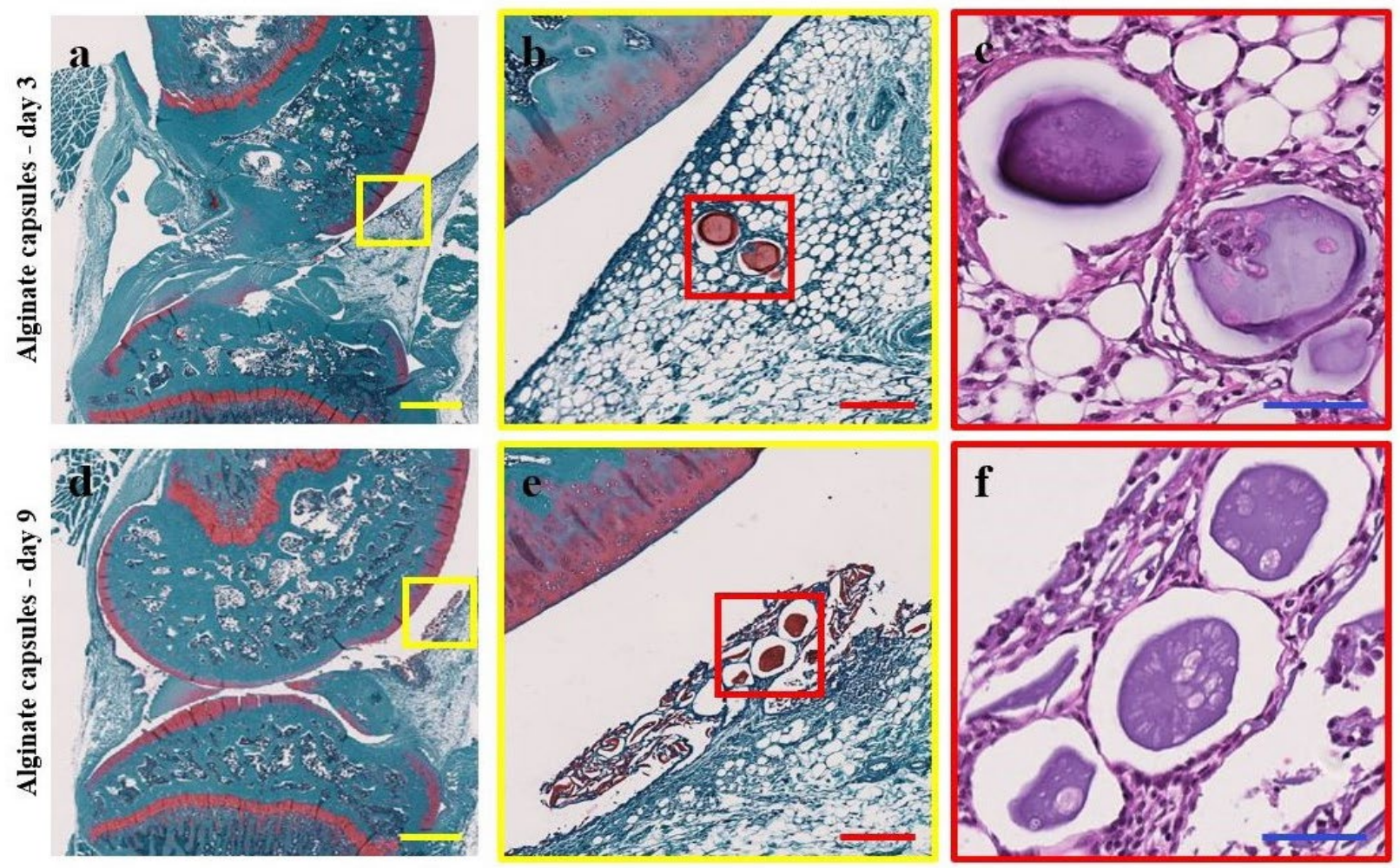

Fig. 4. Representative histological images, single images at three separate magnifications, of full joint histology of rat hind limb knee joints at (a-c) $3 \mathrm{~d}$ and (d-e) $9 \mathrm{~d}$ post injection of encapsulated hMSCs. $(\mathbf{a}, \mathbf{b})$ At day 3, Saf-O-stained joints showed the presence of sodium alginate capsules (stained red) in the infrapatellar fat pad. (c) H\&E staining demonstrated the presence of encapsulated cells in the sodium alginate capsules at this time point. (d,e) Saf-O staining at day 9 displayed intact sodium alginate capsules in the synovial lining of the knee joint space surrounded by remnants of broken down capsules. (f) No encapsulated cells were identifiable at day 9 , as demonstrated by H\&E staining, but lacunae were identifiable appearing either empty or containing cell debris. In each image, the anterior hindlimb is located on the right and the posterior hindlimb on the left. Scale bars: yellow $=1 \mathrm{~mm}$, red $=200 \mu \mathrm{m}$, blue $=60 \mu \mathrm{m}$. 
therapeutic for early stage OA in a pre-clinical rat model.

hMSC encapsulation allows the study of the paracrine signaling properties of these cells independently of direct engraftment, as the capsule provides a mechanical barrier preventing the direct incorporation of the hMSCs into the native tissue (Landázuri et al., 2012). Additionally, encapsulation improves MSC survival, which is critical for the therapeutic efficacy (Landázuri et al., 2012; Leijs et al., 2017; Levit et al., 2013). To study the effects encapsulation would have on viability, retention, proliferation and metabolic state of hMSCs, a bioluminescence-tracking study was performed using luciferase-expressing hMSCs. While encapsulation yielded a statistically significant increase in hMSC bioluminescence, the enhancement of cell bioluminescent signal was not as pronounced as was initially hypothesized. The initial hypothesis was based on a previous work in a rat myocardial infarction model showing a sizable increase in the bioluminescent signal of the encapsulated hMSCs (Levit et al., 2013). Even though Levit et al. (2013) show similar encapsulated hMSC bioluminescent signal detection for approximately $7 \mathrm{~d}$, hMSCs alone yield an attenuated bioluminescence $1 \mathrm{~d}$ post-injection. However, in the present study, the bioluminescence of the non-encapsulated hMSCs $(\sim 7 \mathrm{~d})$ was consistent with a previous report using intra-articular injections
(Horie et al., 2012). Van Buul et al. (2014) show that non-encapsulated hMSCs can survive for up to $14 \mathrm{~d}$ following intra-articular injections in a rat model; however, one key difference is that those results are obtained using allogeneic MSC sources. While many questions remain as to the effects of cell sourcing, particularly the utilization of an allogenic versus xenogeneic source, numerous reports demonstrate decreased cell variability when using xenogeneic $v s$. allogenic cells (Lin et al., 2012; Niemeyer et al., 2008). Furthermore, while previous studies demonstrate beneficial effects of encapsulation on hMSC viability, direct comparison of these reports to the present study was confounded by the various capsule sizes employed and the different sites of delivery employed, as each location could contain different clearance kinetics, mechanical loading and immune response components (Landázuri et al., 2016; Leijs et al., 2017; Levit et al., 2013). In the present study, histological analysis of alginate capsule retention showed the presence of encapsulated cells at day 3 post-injection, consistent with the bioluminescent data, but cells were not identifiable in the capsules at day 9. However, at day 9, the capsules did show open spaces resembling lacunae that were either empty or contained cell debris, suggesting potential cell apoptosis. To confirm that this cell death was not due to technical issues with encapsulation, an in vitro study was performed demonstrating
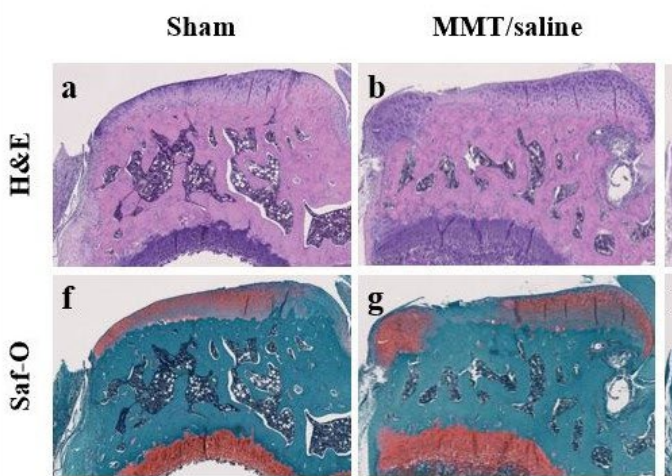

MMT/empty caps
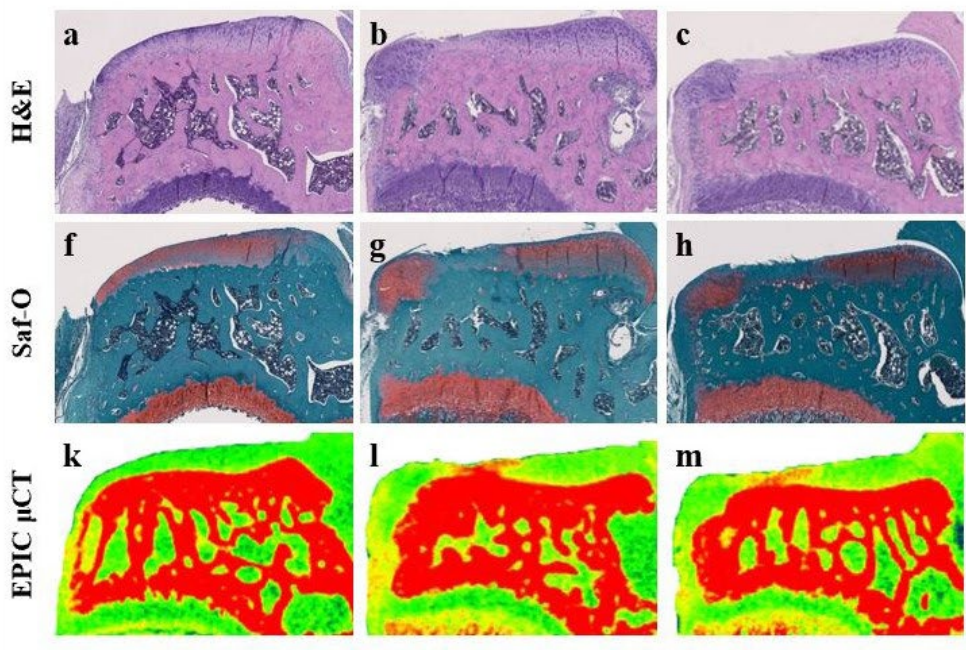

MMT/hMSC
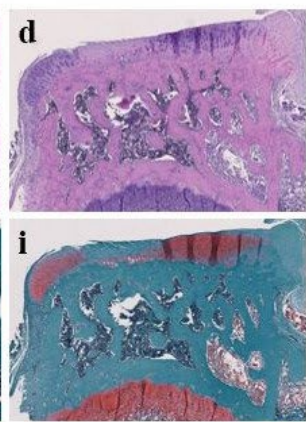

n

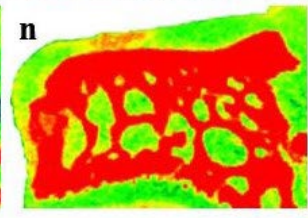

MMT/encap hMSC

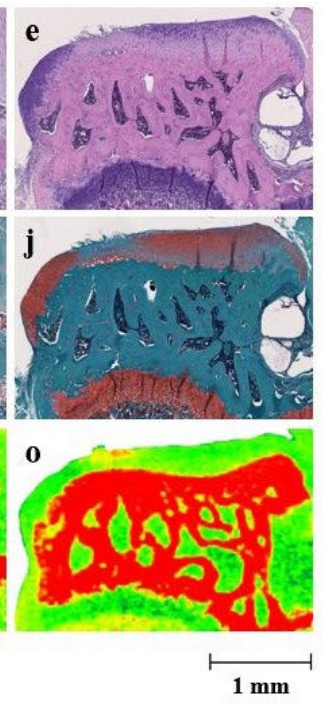

Fig. 5. Representative histology and EPIC- $\mu$ CT coronal images of medial tibial condyle following encapsulated hMSC treatment. (a-e) H\&E- and (f-j) Saf-O-stained MMT joints showed no cartilage damage or osteophyte development in (a,f) sham control; (b-e,g-j) proteoglycan loss, degeneration of cartilaginous surfaces and osteophyte development in all MMT conditions. (k-o) Corresponding EPIC- $\mu$ CT images showed similar disease progression as shown by histology. (k) No cartilage damage was observed in sham control; (1-o) increased areas of cartilage attenuation, specifically in the medial 1/3, was observed in all MMT conditions in addition to osteophyte volumes. Red indicates higher cartilage attenuation, corresponding to lower proteoglycan content. In all images, the medial tibial condyle is located on the left and the lateral tibial condyle on the right. Scale bar (bottom right corner) is universal for all histology and EPIC- $\mu \mathrm{CT}$ representative images. 
encapsulated hMSC survival for up to $35 \mathrm{~d}$. While cell viability is a key component of the therapeutic efficacy of MSC treatments, recent reports suggest that hMSC apoptosis may play a key role in the immunomodulatory properties of these cells (Mamidi et al., 2016; Pigott et al., 2013; Galleu et al., 2017). Overall, while the study demonstrated that hMSC encapsulation slightly enhanced bioluminescent signal in comparison to hMSCs alone, both groups showed no signal by day 9 post-injection. One limitation of this bioluminescent technique was that hMSC viability, retention, proliferation and metabolic state could not be studied independently. Further study will be needed to discriminate the potential individual contributions of each of these cellular properties.

To study the therapeutic effects of encapsulated hMSCs, EPIC- $\mu$ CT analysis was used to quantitatively evaluate changes in articular cartilage structure and composition, osteophyte volumes and subchondral bone morphology. EPIC- $\mu$ CT data showed articular cartilage lower thickness and increased surface roughness in the encapsulated hMSC group in comparison to all other MMT conditions in the early stages of $\mathrm{OA}$, as the therapeutic was delivered at the time of a traumatic injury to the joint but before OA had developed. Swelling of the articular cartilage precedes fibrillation development and augmented surface roughness in the early stages of OA (Bertrand et al., 2010). Therefore, encapsulated hMSCs showed a chondroprotective role in early OA as they were associated with both attenuated swelling of articular cartilage and surface roughness as compared to the control groups. These findings were consistent with pre-clinical OA models showing decreased cartilage degeneration and a cartilage protective effect in MSC-treated animals (Diekman et al., 2013; Murphy et al., 2003; Toghraie et al., 2012). Furthermore, the chondroprotective role of MSCs in OA is reported in ex vivo human tissue samples with MSC treatments

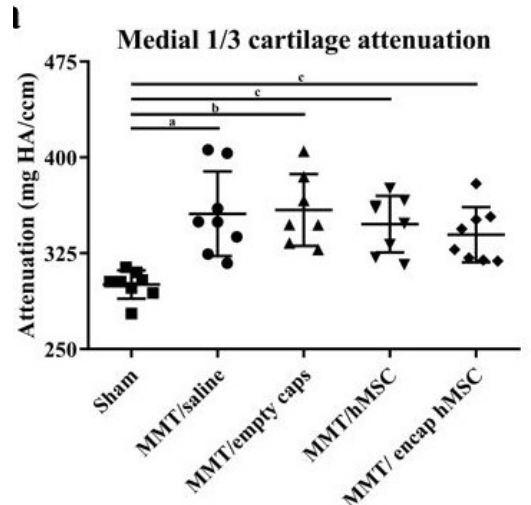

b

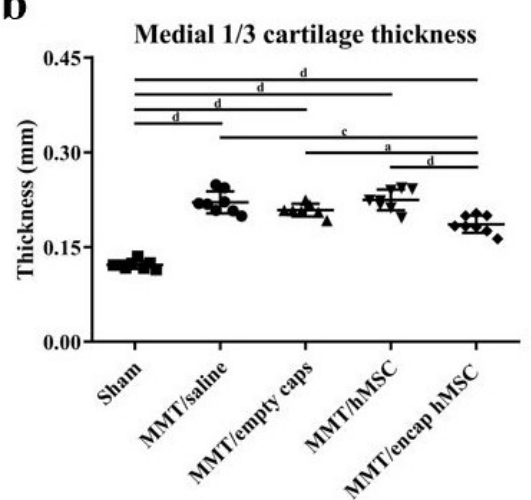

c

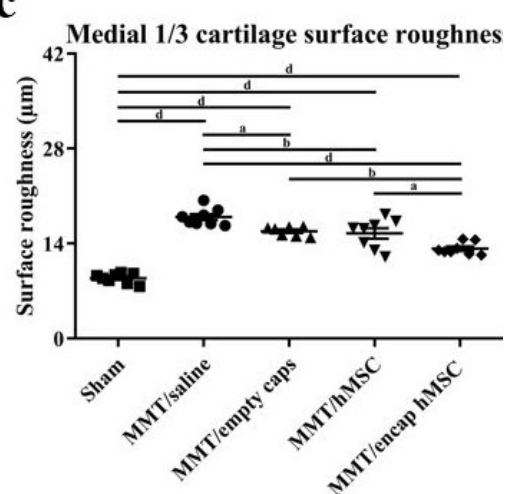

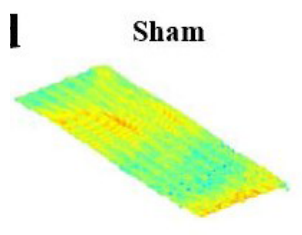

e MMT/saline

f MMT/empty caps

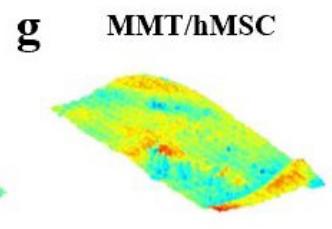

h MMT/encap hMSC
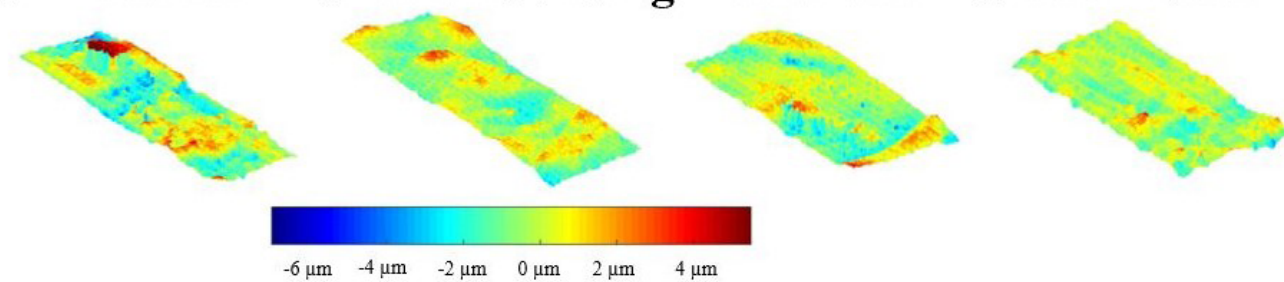

Fig. 6. EPIC- $\mu \mathrm{CT}$ quantification of articular cartilage structure and composition in the medial $1 / 3$ of the medial articular cartilage in MMT joints treated with encapsulated hMSCs. (a) Cartilage attenuation was significantly increased in all MMT conditions as compared to sham control. (b) Cartilage thickness was significantly increased in all MMT conditions as compared to sham control; encapsulated hMSCs attenuated increases in cartilage thickness as compared to all other MMT conditions. (c) Cartilage surface roughness was significantly increased in all MMT conditions as compared to sham control; saline augmented cartilage surface roughness as compared to all other MMT conditions; encapsulated hMSCs attenuated increases in cartilage surface roughness as compared to all other MMT conditions. (d-h) Representative differential surfaces illustrating surface roughness of the medial $1 / 3$ of the articular cartilage of the medial tibial condyle. Differential maps were generated by subtracting individual 3D polynomial surfaces from the corresponding cartilage surface renderings. In these images, red indicates points that are more proximal and blue points that are further distal. (e-h) Qualitative analysis of the cartilage surfaces demonstrated increased surface roughness in all MMT conditions relative to the sham control in (d). (h) The representative surface for encap hMSCs also displayed decreased surface roughness when compared to all other MMT conditions (e-g). Data presented as mean \pm SD. $n=7 /$ group for MMT/empty caps; $n=8 /$ group for all other conditions. ${ }^{\mathrm{a}} p<0.05 ;{ }^{\mathrm{b}} p \leq 0.01 ;{ }^{c} p \leq 0.001 ;{ }^{\mathrm{d}} p \leq 0.0001$. 
yielding a significant reduction in the expression of fibrotic and hypertrophic markers (Maumus et al., 2013). The data from the present study showed that independently of direct engraftment, hMSCs could exert a chondroprotective therapeutic effect in early OA, reinforcing the significance of the MSC paracrine signaling properties in OA treatment. One limitation of the study was the lack of secretome analysis on the hMSCs following injection; the successful recovery of cells following intra-articular delivery is technically challenging but would provide substantial information if a reliable approach would be used. This limitation motivates further study into the identification of factors being released by hMSCs when stimulated by the osteoarthritic environment. hMSC paracrine signaling properties show great therapeutic potential in protecting the joint cartilaginous surfaces in early OA but the secondary effects of these hMSC communication mechanisms must also be considered in assessing overall therapeutic efficacy.

OA-associated pathologies are not limited to phenotypic changes in the articular cartilage but can also affect the surrounding tissue, through the formation of osteophytes, synovial hyperplasia and subchondral bone sclerosis (Loeser et al., 2012). Osteophytes are clinically defined as bony outgrowths - containing a fibrocartilaginous cap that forms on the margins of weight-bearing joints - and are a staple of OA development (van der Kraan and van der Berg, 2007). EPIC- $\mu$ CT quantitative analysis showed an increase in mineralized osteophyte volume for
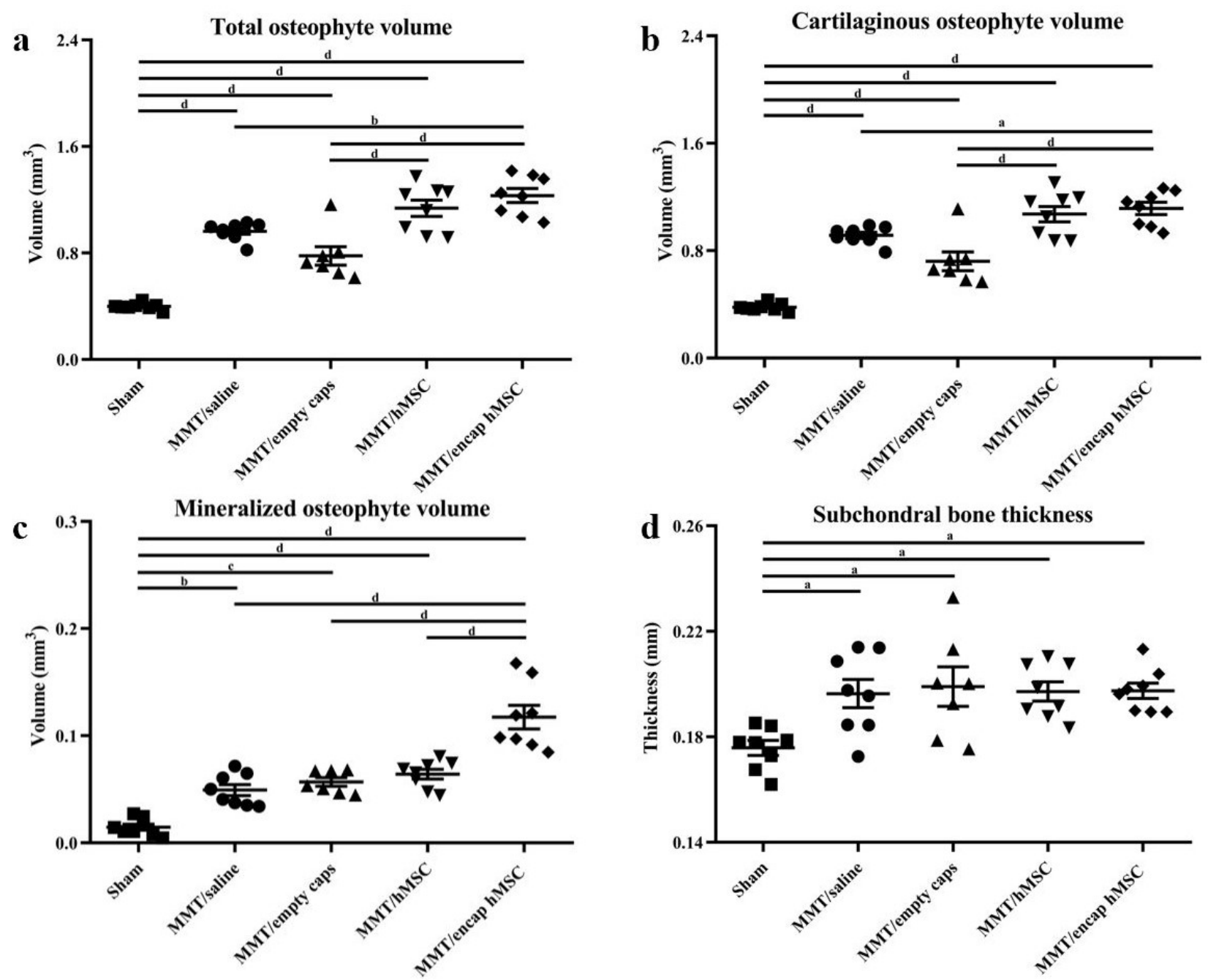

Fig. 7. EPIC- $\mu \mathrm{CT}$ quantification of osteophyte formation and subchondral bone morphology of medial side of MMT joints treated with encapsulated hMSCs. (a) Total osteophyte volume was significantly increased in all MMT conditions as compared to sham control; encapsulated hMSCs augmented osteophyte volumes as compared to saline and empty capsule conditions. (b) Cartilaginous osteophyte volumes yielded the same respective differences among groups as the total osteophyte volume parameter. (c) Mineralized osteophyte volume was significantly increased in all MMT conditions as compared to sham control; encapsulated hMSCs augmented mineralized osteophyte volumes as compared to all other MMT conditions. (d) Subchondral bone thickness was significantly increased in all MMT conditions as compared to sham control. Data presented as mean \pm SD. $n=7 /$ group MMT/empty caps; $n=8 /$ group for all other conditions. ${ }^{\mathrm{a}} p<0.05 ;{ }^{\mathrm{b}} p \leq 0.01 ;{ }^{\mathrm{c}} p \leq 0.001 ;{ }^{\mathrm{d}} p \leq 0.0001$. 
the encapsulated hMSC group in comparison to all control groups. Additionally, cartilaginous osteophyte volumes were larger for encapsulated hMSCs in comparison to all groups, except the hMSC group. The effects that MSCs can have on osteophyte formation remains largely unknown. Osteophytes in general are understudied and their role and function remain poorly understood in OA. While the formation of osteophytes is hypothesized to be a compensatory mechanism in early OA, late stage osteophytes may be associated with further stages of disease progression and symptoms (Muraki et al., 2011; Serban et al., 2016; van der Berg, 1999). Even though studies as to the mechanical role of osteophytes in the knee are lacking, the function of osteophytes in the vertebral bodies of the human spines is reported (Al-Rawahi et al., 2011). Vertebral body osteophytes increase motion segment resistance to both bending and compression forces, suggesting that osteophytes' formation reverses the mechanical stimuli that cause them to form, in a possible compensatory and protective role (Al-Rawahi et al., 2011). Clinically, in late stage knee OA, osteophyte formation strongly correlates with pain level and patient quality of life (Muraki et al., 2011; Serban et al., 2016; van der Berg, 1999); however, cartilage damage (and not osteophytes) is the strongest predictor of pain in these same late stage OA patients (Serban et al., 2016). Studies isolating the associated pain levels of these late stage pathologies, independent of one another, are limited (Serban et al., 2016). In addition to these studies, Kirkley et al. (2008) demonstrate that arthroscopic removal of osteophytes brings no additional clinical benefits. The clinical implications of osteophytes are not well characterized in early $\mathrm{OA}$, as osteophyte formation usually presents (or is at least identified and reported) in the latter stages of the disease, when these structures are augmented and more radiographically identifiable. While the present study did not analyze late stage OA, the results warrant further study.

Even though many questions remain as to the mechanism of osteophyte formation, MSCs are implicated as a major player in their development (van der Kraan and van der Berg, 2007). Osteophytes develop from the marginal tissue periosteum surrounding subchondral cortical bone, where endogenous MSCs are stimulated to proliferate by various mechanical and biochemical cues (van der Kraan and van der Berg, 2007). Then, cells within the developing osteophyte undergo chondrogenesis and differentiate into mature hypertrophic chondrocytes (van der Kraan and van der Berg, 2007). Following the development of this cartilaginous tissue, vascular invasion occurs and chondrocytes are replaced by osteoblasts to form bone and marrow cavities (van der Kraan and van der Berg, 2007). This developmental process is similar to the endochondral ossification in bone growth plates, however, the exact mechanism has yet to be elucidated. Fully developed osteophytes integrate with the subchondral bone and retain an outer covering of cartilage, expanding the original cartilage surface of the joint (van der Kraan and van der Berg, 2007). The implication of MSCs as a major player in osteophyte development speaks to the potential role hMSC paracrine factors may have in augmenting osteophyte formation. Numerous paracrine factors that are implicated in osteophyte development are identified in the hMSC secretome, including fibroblast growth factor (FGF)-9, TGF- $\beta$, bone morphogenic protein (BMP)-2 and insulin-like growth factor (IGF)-1 (Davidson et al., 2007; Okazaki et al., 1999; van der Kraan and van der Berg, 2007; Zhou et al., 2016). While many questions remain about the role of osteophytes, the results of the present study should warrant consideration and further investigation when using hMSCs in patients.

Quantitative EPIC- $\mu \mathrm{CT}$ analysis of subchondral bone thickness showed an increase in subchondral bone thickness for all MMT conditions in comparison to the sham control. This increase in thickness was evidence of increased sclerosis (abnormal hardening), which is a hallmark of OA development (Radin et al., 1972). However, no differences were detected between any of the MMT conditions, indicating a lack of enhancement or of a protective effect of subchondral bone sclerosis by encapsulated hMSCs. These results contrast previous studies reporting a protective effect of MSCs on subchondral bone in the form of decreased mineralization and subsequent reduction in sclerosis (thickening) (Kim et al., 2014; Murphy et al., 2003). Changes in the synovium were not assessed in the current study since significant differences in the synovium, between sham and MMT animals, do not present in this model until later time points (Kloefkorn et al., 2017). Collectively, the results of the study showed that hMSC paracrine communication yielded a chondroprotective role on articular cartilage in early OA but also augmented osteophyte formations.

The hMSC group, in comparison to the encapsulated hMSC group, did not yield the same early chondroprotective effect and did not yield significantly larger mineralized osteophyte volumes than the other study groups, as measured by EPIC- $\mu \mathrm{CT}$ analysis. The results of the luciferasetracking study suggested that the increased hMSC bioluminescence played a minor role in the therapeutic efficacy of hMSCs, as encapsulation did not enhance bioluminescent activity as markedly as was initially hypothesized. It is possible that encapsulation may also affect the paracrine signaling properties of hMSCs and further contribute to the results yielded in the EPIC- $\mu \mathrm{CT}$ analyses. However, a limitation of the study was the inability to delineate between the effects of hMSC bioluminescence (viability, retention, proliferation and metabolic state) and the paracrine signaling properties of these cells, as these could not be specifically isolated and studied independently of one another. A further limitation was the inability to successfully recover encapsulated hMSCs from the knee joint. For both the encapsulated and 
non-encapsulated groups, the same batch of cells were used for intra-articular injections, with both containing the same initial secretome factors. In vitro, encapsulation affects the MSC secretome, with certain factors being differently affected in comparison to MSC monolayer cultures (Landázuri et al., 2016; Stucky et al., 2015). Encapsulation upregulates prostaglandin $\mathrm{E}_{2}\left(\mathrm{PGE}_{2}\right)$, which reduces the levels of the pro-inflammatory cytokine $\mathrm{TNF} \alpha$, implicated in the breakdown of cartilage in OA (Kunisch et al., 2016; Stucky et al., 2015). However, the study by Stucky et al. (2015) is unable to delineate between the individual effects of the alginate and the 3D culture environment within the micro-capsule and the role each of these had in the study results. Furthermore, encapsulation of hMSCs upregulates numerous factors, including bFGF, phosphatidylinositol-glycan biosynthesis class F protein (PIGF) and vascular endothelial growth factor (VEGF), and downregulates the hepatocyte growth factor (HGF), among others, in comparison to non-encapsulated cells (Landázuri et al., 2012). This potential for alterations in paracrine secretory activity might also account for the differences noted in the present study. Additionally, while the intercellular signaling properties of extracellular vesicles are an important mediator in the therapeutic efficacy of MSCs in OA, these structures are unable to readily diffuse through the capsules employed in the present study (Cosenza et al., 2017). The two main types of extracellular vesicles, exosomes and microvesicles, which maintain sizes of 50-150 nm and $50-500 \mathrm{~nm}$, respectively, exceed the $40 \mathrm{~nm}$ pore size of the capsules previously established (molecular diameter IgG $40 \mathrm{~nm}$ ) (Landázuri et al., 2012; van Niel et al., 2018). The potential effects encapsulation has on enhanced bioluminescent activity and varied paracrine secretory activity are important observations. This motivates further study of the effects encapsulation has on the secretory profiles of hMSC s and hMSCs paracrine communication and of hMSCs and encapsulation therapeutic efficacy.

With the substantial and increasing burden of OA and limited treatment options outside of temporary symptomatic relief, there is a critical need for the development of disease-modifying therapeutics. MSCs show great promise but questions remain as it relates to their mechanism of action. The present study showed that hMSC could exert a chondroprotective therapeutic effect through paracrine signaling, independent of direct engraftment, as encapsulated hMSCs yielded an early protective role on articular cartilage in OA. Furthermore, the study showed the effects hMSCs, through their paracrine signaling properties, could have on osteophyte formation, as encapsulated hMSCs increased osteophyte volumes. These augmented tissue volumes are especially relevant in clinical applications as many clinical trials are currently ongoing but the effects of osteophyte development have not been investigated so far. Further study of the efficacy of these encapsulated
hMSCs on OA progression after the disease has developed will have clinical relevance, as OA is not diagnosed clinically until patients present with augmented OA phenotypes in later stage OA.

\section{Conclusions}

Encapsulation of bone marrow hMSCs with sodium alginate allowed for the assessment of the paracrine signaling properties of these cells, by preventing their direct engraftment into the native tissue. The paracrine mechanisms of these hMSCs showed a chondroprotective role for articular cartilage in early stage post-traumatic OA, attenuating increases in articular cartilage swelling and surface roughness. However, in addition to these early protective effects, cartilaginous and mineralized osteophyte volumes were enhanced. This early cartilage protective effect was consistent with previous reports of MSC therapeutics for OA, suggesting that direct engraftment of MSCs might not be necessary for the therapeutic benefit of an MSC injection for OA. However, the effects MSCs could have on augmenting osteophyte formations was a new observation and warrants consideration and further investigation as MSC therapies are currently being used clinically. Overall, the study demonstrated that the paracrine signaling properties of hMSCs, alone, could exert a chondroprotective therapeutic effect in early stage OA.

\section{Acknowledgements}

This work was supported in part by VA (SPiRE) Grant I21RX002372-01A1 from the United States (U.S.) Department of Veterans Affairs Rehabilitation Research and Development Service. The research was also supported in part by the DOD PRMRP Grant PR171379 and PHS Grant UL1TR000454 from the Clinical and Translational Science Award Program, National Institutes of Health, National Center for Advancing Translational Sciences. The authors would like to thank Mila Friedman for the histological analyses and Colleen Oliver for her support in the animal study.

\section{Dislaimer}

The contents do not represent the views of the U.S. Department of Veterans Affairs or the United States Government.

\section{References}

Allen AB, Gazit Z, Su S, Stevens HY, Guldberg RE (2014) In vivo bioluminescent tracking of 
mesenchymal stem cells within large hydrogel constructs. Tissue Eng Part C Methods 20: 806-816.

Al-Rawahi M, Luo J, Pollintine P, Dolan P, Adams MA (2011) Mechanical function of vertebral body osteophytes, as revealed by experiments on cadaveric spines. Spine 36: 770-777.

Bendele AM (2001) Animal models of osteoarthritis. J Musculoskelet Neuronal Interact 1: 363-376.

Bertrand J, Cromme C, Umlauf D, Frank S, Pap T (2010) Molecular mechanisms of cartilage remodeling in osteoarthritis. Int J Biochem Cell Biol 42: 1594-1601.

Campeau E, Ruhl VE, Rodier F, Smith CL, Rahmberg BL, Fuss JO, Campisi J, Yaswen P, Cooper PK, Kaufman PD (2009) A versatile viral system for expression and depletion of proteins in mammalian cells. PLoS One 4: e6529.

Choi H, Lee RH, Bazhanov N, Oh JY, Prockop DJ (2011) Anti-inflammatory protein TSG-6 secreted by activated MSCs attenuates zymosan-induced mouse peritonitis by decreasing TLR2/NF- $\kappa B$ signaling in resident macrophages. Blood 118: 330-338.

Cisternas MG, Murphy L, Sacks JJ, Solomon DH, Pasta DJ, Helmick CG (2016) Alternative methods for defining osteoarthritis and the impact on estimating prevalence in a US population-based survey. Arthritis Care Res 68: 574-80.

Cosenza S, Ruiz M, Toupet K, Jorgensen C, Noël D (2017) Mesenchymal stem cells derived exosomes and microparticles protect cartilage and bone from degradation in osteoarthritis. Sci Rep 7: 16214. DOI: 10.1038/s41598-017-15376-8.

Davidson ENB, Vitters EL, van Beuningen $\mathrm{HM}$, van de Loo FAJ, van den Berg WB, van der Kraan PM (2007) Resemblance of osteophytes in experimental osteoarthritis to transforming growth factor $\beta$-induced osteophytes: limited role of bone morphogenetic protein in early osteoarthritic osteophyte formation. Arthritis Rheum 56: 4065-4073.

Diekman BO, Wu C-L, Louer CR, Furman BD, Huebner JL, Kraus VB, Olson SA, Guilak F (2013) Intra-articular delivery of purified mesenchymal stem cells from C57BL/6 or MRL/MpJ superhealer mice prevents posttraumatic arthritis. Cell Transplant 22: 1395-1408.

Farrell E, Fahy N, Ryan AE, Flatharta CO, O'Flynn L, Ritter T, Murphy JM (2016) VIL-10-overexpressing human MSCs modulate naïve and activated T lymphocytes following induction of collagenaseinduced osteoarthritis. Stem Cell Res Ther 7: 74. DOI: 10.1186/s13287-016-0331-2.

Galleu A, Riffo-Vasquez Y, Trento C, Lomas C, Dolcetti L, Cheung TS, Von Bonin M, Barbieri L, Halai K, Ward S, Weng L, Chakraverty R, Lombardi G, Watt F, Orchard K, Marks D, Apperley J, Bornhauser M, Walczak H, Bennett C, Dazzi F (2017) Apoptosis in mesenchymal stromal cells induces in vivo recipientmediated immunomodulation. Sci Transl Med 9: 1-11.

Grigolo B, Lisignoli G, Desando G, Cavallo C, Marconi E, Tschon M, Giavaresi G, Fini M, Giardino R, Facchini A (2009) Osteoarthritis treated with mesenchymal stem cells on hyaluronan-based scaffold in rabbit. Tissue Eng Part C Methods 15: 647-658.

Hatsushika D, Muneta T, Horie M, Koga H, Tsuji K, Sekiya I (2013) Intraarticular injection of synovial stem cells promotes meniscal regeneration in a rabbit massive meniscal defect model. J Orthop Res 31: 13541359.

Horie M, Choi H, Lee RH, Reger RL, Ylostalo J, Muneta T, Sekiya I, Prockop DJ (2012) Intra-articular injection of human mesenchymal stem cells (MSCs) promote rat meniscal regeneration by being activated to express Indian hedgehog that enhances expression of type II collagen. Osteoarthr Cartil 20: 1197-1207.

Janusz MJ, Bendele AM, Brown KK, Taiwo YO, Hsieh L, Heitmeyer SA (2002) Induction of osteoarthritis in the rat by surgical tear of the meniscus: inhibition of joint damage by a matrix metalloproteinase inhibitor. Osteoarthr Cartil 10: 785-791.

Kim JE, Lee SM, Kim SH, Tatman P, Gee AO, Kim D-H, Lee KE, Jung Y, Kim SJ (2014) Effect of selfassembled peptide-mesenchymal stem cell complex on the progression of osteoarthritis in a rat model. Int J Nanomedicine 9 Suppl 1: 141-157.

Kintscher U, Wakino S, Bruemmer D, Goetze S, Graf K, Hsueh WA, Law RE (2002) TGF-beta(1) induces peroxisome proliferator-activated receptor gamma1 and gamma2 expression in human THP-1 monocytes. Biochem Biophys Res Commun 297: 794799.

Kirkley A, Birmingham TB, Litchfield RB, Giffin JR, Willits KR, Wong CJ, Feagan BG, Donner A, Griffin SH, D’ Ascanio LM, Pope JE, Fowler PJ (2008) A randomized trial of arthroscopic surgery for osteoarthritis of the knee. N Engl J Med 359: 10971107.

Kloefkorn HE, Allen KD (2017) Quantitative histological grading methods to assess subchondral bone and synovium changes subsequent to medial meniscus transection in the rat. Connect Tissue Res 58: 373-385.

Kunisch E, Kinne RW, Alsalameh RJ, Alsalameh S (2016) Pro-inflammatory IL-1beta and/or TNFalpha up-regulate matrix metalloproteases- 1 and -3 mRNA in chondrocyte subpopulations potentially pathogenic in osteoarthritis: in situ hybridization studies on a single cell level. Int J Rheum Dis 19: 557566.

Kunzmann S, Wright JR, Steinhilber W, Kramer BW, Blaser K, Speer CP, Schmidt-Weber C (2006) TGF-beta1 in SP-A preparations influence immune suppressive properties of SP-A on human CD4+ T lymphocytes. Am J Physiol Lung Cell Mol Physiol 291: L747-756.

Landázuri N, Levit RD, Joseph G, Ortega-Legaspi JM, Flores CA, Weiss D, Sambanis A, Weber CJ, Safley SA, Taylor WR (2012) Alginate microencapsulation of human mesenchymal stem cells as a strategy to enhance paracrine-mediated vascular recovery after hindlimb ischaemia. J Tissue Eng Regen Med 10: 222232. 
Leijs M, Villafuertes E, Haeck J, Koevoet W, Fernandez-Gutierrez B, Hoogduijn M, Verhaar J, Bernsen M, van Buul G, van Osch G (2017) Encapsulation of allogeneic mesenchymal stem cells in alginate extends local presence and therapeutic function. Eur Cell Mater 33: 43-58.

Levit RD, Landázuri N, Phelps EA, Brown ME, Garcia AJ, Davis ME, Joseph G, Long R, Safley SA, Suever JD, Lyle AN, Weber CJ, Taylor WR (2013) Cellular encapsulation enhances cardiac repair. J Am Heart Assoc 2: e000367. DOI: 10.1161/ JAHA.113.000367.

Lin CS, Lin G, Lue TF (2012) Allogeneic and xenogeneic transplantation of adipose-derived stem cells in immunocompetent recipients without immunosuppressants. Stem Cells Dev 21: 2770-2778.

Liu X, Duan B, Cheng Z, Jia X, Mao L, Fu H, Che Y, Ou L, Liu L, Kong D (2011) SDF-1/CXCR4 axis modulates bone marrow mesenchymal stem cell apoptosis, migration and cytokine secretion. Protein Cell 2: 845-854.

Loeser RF, Goldring SR, Scanzello CR, Goldring MB (2012) Osteoarthritis: a disease of the joint as an organ. Arthritis Rheum 64: 1697-1707.

Mamidi MK, Das AK, Zakaria Z, Bhonde R (2016) Mesenchymal stromal cells for cartilage repair in osteoarthritis. Osteoarthr Cartil 24: 1307-1316.

Martel-Pelletier J (2004) Pathophysiology of osteoarthritis. Osteoarthr Cartil 12 Suppl A: S31-3.

Maumus M, Manferdini C, Toupet K, Peyrafitte J-A, Ferreira R, Facchini A, Gabusi E, Bourin P, Jorgensen C, Lisignoli G, Noël D (2013) Adipose mesenchymal stem cells protect chondrocytes from degeneration associated with osteoarthritis. Stem Cell Res 11: 834-844.

Messier SP, Loeser RF, Miller GD, Morgan TM, Rejeski WJ, Sevick MA, Ettinger WH, Pahor M, Williamson JD (2004) Exercise and dietary weight loss in overweight and obese older adults with knee osteoarthritis: the arthritis, diet, and activity promotion trial. Arthritis Rheum 50: 1501-1510.

Muraki S, Oka H, Akune T, En-Yo Y, Yoshida M, Suzuki T, Yoshida H, Ishibashi H, Tokimura F, Yamamoto S, Nakamura K, Kawaguchi H, Yoshimura N (2011) Independent association of joint space narrowing and osteophyte formation at the knee with health-related quality of life in Japan: a cross-sectional study. Arthritis Rheum 63: 3859-3864.

Murphy JM, Fink DJ, Hunziker EB, Barry FP (2003) Stem cell therapy in a caprine model of osteoarthritis. Arthritis Rheum 48: 3464-3474.

Namba A, Aida Y, Suzuki N, Watanabe Y, Kawato T, Motohashi M, Maeno M, Matsumura H, Matsumoto M (2007) Effects of IL-6 and soluble IL-6 receptor on the expression of cartilage matrix proteins in human chondrocytes. Connect Tissue Res 48: 263270.

Niemeyer P, Vohrer J, Schmal H, Kasten P, Fellenberg J, Suedkamp NP, Mehlhorn AT (2008) Survival of human mesenchymal stromal cells from bone marrow and adipose tissue after xenogenic transplantation in immunocompetent mice. Cytotherapy 10: 784-795.

Okazaki K, Jingushi S, Ikenoue T, Urabe K, Sakai H, Ohtsuru A, Akino K, Yamashita S, Nomura S, Iwamoto Y (1999) Expression of insulin-like growth factor I messenger ribonucleic acid in developing osteophytes in murine experimental osteoarthritis and in rats inoculated with growth hormonesecreting tumor. Endocrinology 140: 4821-4830.

Orive G, Hernández RM, Gascón AR, Calafiore R, Chang TMS, Vos P De, Hortelano G, Hunkeler D, Lacík I, Shapiro AMJ, Pedraz JL (2003) Cell encapsulation: promise and progress. Nat Med 9: 104-107.

Palmer AW, Guldberg RE, Levenston ME (2006) Analysis of cartilage matrix fixed charge density and three-dimensional morphology via contrast-enhanced microcomputed tomography. Proc Natl Acad Sci 103: 19255-19260.

Pers Y-M, Ruiz M, Noël D, Jorgensen C (2015) Mesenchymal stem cells for the management of inflammation in osteoarthritis: state of the art and perspectives. Osteoarthr Cartil 23: 2027-2035.

Pigott JH, Ishihara A, Wellman ML, Russell DS, Bertone AL (2013) Investigation of the immune response to autologous, allogeneic, and xenogeneic mesenchymal stem cells after intra-articular injection in horses. Vet Immunol Immunopathol 156: 99-106.

Radin EL, Paul IL, Rose RM (1972) Role of mechanical factors in pathogenesis of primary osteoarthritis. Lancet 299: 519-522.

Reece DS, Thote T, Lin ASP, Willett NJ Guldberg RE (2017) Contrast enhanced $\mu C T$ imaging of early articular changes in a pre-clinical model of osteoarthritis. Osteoarthr Cartil 26: 118-127.

Sato M, Uchida K, Nakajima H, Miyazaki T, Guerrero A, Watanabe S, Roberts S, Baba H (2012) Direct transplantation of mesenchymal stem cells into the knee joints of hartley strain guinea pigs with spontaneous osteoarthritis. Arthritis Res Ther 14: R31. DOI: $10.1186 / a r 3735$.

Saulnier N, Viguier E, Perrier-Groult E, Chenu C, Pillet E, Roger T, Maddens S, Boulocher C (2015) Intra-articular administration of xenogeneic neonatal mesenchymal stromal cells early after meniscal injury down-regulates metalloproteinase gene expression in synovium and prevents cartilage degradation in a rabbit model of osteoarthritis. Osteoarth Cartil 23: 122-133.

Schmitz N, Laverty S, Kraus VB, Aigner T (2010) Basic methods in histopathology of joint tissues. Osteoarthr Cartil 18: S113-S116.

Serban O, Porojan M, Deac M, Cozma F, Solomon C, Lenghel M, Micu M, Fodor D (2016) Pain in bilateral knee osteoarthritis - correlations between clinical examination, radiological, and ultrasonographical findings. Med Ultrason 18: 318-325.

Shin EY, Wang L, Zemskova M, Deppen J, Xu K, Strobel F, García AJ, Tirouvanziam R, Levit RD (2018) 
Adenosine production by biomaterial supported mesenchymal stromal cells reduces the innate inflammatory response in myocardial ischemia/ reperfusion injury. J Am Heart Assoc 7: e006949. DOI: 10.1161/JAHA.117.006949.

Stucky EC, Schloss RS, Yarmush ML, Shreiber DI (2015) Alginate micro-encapsulation of mesenchymal stromal cells enhances modulation of the neuroinflammatory response. Cytotherapy 17: 1353-1364.

ter Huurne M, Schelbergen R, Blattes R, Blom A, de Munter W, Grevers LC, Jeanson J, Noël D, Casteilla L, Jorgensen C, van den Berg W, van Lent PLEM (2012) Antiinflammatory and chondroprotective effects of intraarticular injection of adipose-derived stem cells in experimental osteoarthritis. Arthritis Rheum 64: 3604-3613.

Thote T, Lin ASP, Raji Y, Moran S, Stevens HY, Hart M, Kamath R V, Guldberg RE, Willett NJ, Coulter WH, Woodruff GW (2013) Localized 3D analysis of cartilage composition and morphology in small animal models of joint degeneration. Osteoarthr Cartil 21: 1132-1141.

Toghraie F, Razmkhah M, Gholipour MA, Faghih Z, Chenari N, Torabi Nezhad S, Nazhvani Dehghani S, Ghaderi A (2012) Scaffold-free adipose-derived stem cells (ASCs) improve experimentally induced osteoarthritis in rabbits. Arch Iran Med 15: 495-499.

Van Buul GM, Siebelt M, Leijs MJC, Bos PK, Waarsing JH, Kops N, Weinans H, Verhaar J, Bernsen M, Van Osch GJVM (2014) Mesenchymal stem cells reduce pain but not degenerative changes in a monoiodoacetate rat model of osteoarthritis. J Orthop Res 32: 1167-1174.

van der Berg WB (1999) Osteophyte formation in osteoarthritis. Osteoarthr Cartil 7: 333.

van der Kraan PM, van den Berg WB (2007) Osteophytes: relevance and biology. Osteoarthr Cartil 15: 237-244.

van Niel G, D'Angelo G, Raposo G (2018) Shedding light on the cell biology of extracellular vesicles. Nat Rev Mol Cell Biol 19: 213-228.

Vos T, Global Burden of Disease Study 2010 Collaborators (2012) Years lived with disability (YLDs) for 1160 sequelae of 289 diseases and injuries 1990-2010: a systematic analysis for the global burden of disease study 2010. Lancet 380: 2163-2196.

Willett NJ, Thote T, Hart M, Moran S, Guldberg RE, Kamath R V, Woodruff GW (2016) Quantitative pre-clinical screening of therapeutics for joint diseases using contrast enhanced micro-computed tomography. Osteoarthr Cartil 24: 1604-1612.

Willett NJ, Thote T, Lin AS, Moran S, Raji Y, Sridaran S, Stevens HY, Guldberg RE (2014) Intraarticular injection of micronized dehydrated human amnion/chorion membrane attenuates osteoarthritis development. Arthritis Res Ther 16: R47. DOI: 10.1186/ar4476.

Xie L, Lin ASP, Guldberg RE, Levenston ME (2010) Nondestructive assessment of sGAG content and distribution in normal and degraded rat articular cartilage via EPIC-microCT. Osteoarthritis Cartilage 18: 65-72.

Yu SP, Hunter DJ (2016) Intra-articular therapies for osteoarthritis. Expert Opin Pharmacother 17: 2057-2071.

Zhou S, Wang Z, Tang J, Li W, Huang J, Xu W, Luo F, Xu M, Wang J, Wen X, Chen L, Chen H, Su N, Shen Y, Du X, Xie Y, Chen L (2016) Exogenous fibroblast growth factor 9 attenuates cartilage degradation and aggravates osteophyte formation in post-traumatic osteoarthritis. Osteoarthritis Cartilage 24: 2181-2192.

\section{Web Reference}

1. https:/clinicaltrials.gov/ct2/home [11-10-2017].

\section{Discussion with Reviewers}

Gerben van Buul: What is the effect of alginate on inflammation?

Authors: The immunogenicity of alginate capsules is influenced by alginate composition, method of synthesis and, in some cases, animal species. UP-LVG, bound to barium and lacking an outer polycationic membrane, as was the case in the present study, has better biocompatibility in vivo (Figliuzzi et al., 2006; Safley et al., 2008, additional references). Furthermore, microcapsules are more biocompatible in rodents, when compared to other species (Vaithilingam et al., 2011, additional reference). It is also important to note that the empty capsule group was used as a control for any potential effects of the alginate capsules.

Mary Murphy: The increases in osteophyte formation observed are somewhat troubling; do the authors have any opinion on whether this would be the same outcome obtained when using syngeneic or even allogeneic MSCs? Additionally, is EPIC- $\mu$ CT previously shown to be associated with increased sensitivity for detection of osteophytes?

Authors: Increases in osteophyte volumes with encapsulated hMSC treatment also came as a surprise as no previous study reports this finding. However, MSCs can secrete numerous growth factors associated with endochondral ossification (Li et al., 2016, additional reference). No other sourced encapsulated MSCs (allogeneic or syngeneic) were used in the MMT model and due to the scarcity of previous literature reporting these findings it is not possible to speculate on how alternatively sourced MSCs may impact these findings. It will be critically important for future studies to investigate this exact question. Additionally, EPIC- $\mu \mathrm{CT}$ demonstrates increased sensitivity in analyzing osteophyte parameters, relative to the gold standard in the musculoskeletal field, histopathology (Willett et al., 2016). 


\section{Additional References}

Figliuzzi M, Plati T, Cornolti R, Adobati F, Fagiani A, Rossi L, Remuzzi G, Remuzzi A (2006) Biocompatibility and function of microencapsulated pancreatic islets. Acta Biomater 2: 221-227.

Li C, Li G, Liu M, Zhou T, Zhou H (2016) Paracrine effect of inflammatory cytokine-activated bone marrow mesenchymal stem cells and its role in osteoblast function. J Biosci Bioeng 121: 213-219.

Safley SA, Cui H, Cauffiel S, Tucker-Burden C, Weber CJ (2008) Biocompatibility and immune acceptance of adult porcine islets transplanted intraperitoneally in diabetic NOD mice in calcium alginate poly-L-lysine microcapsules versus barium alginate microcapsules without poly-L-lysine. J Diabetes Sci Technol 2: 760-767.

Vaithilingam V, Kollarikova G, Qi M, Lacik I, Oberholzer J, Guillemin GJ, Tuch BE (2011) Effect of prolonged gelling time on the intrinsic properties of barium alginate microcapsules and its biocompatibility. J Microencapsul 28: 499-507.

Editor's note: The Scientific Editor responsible for this paper was Mauro Alini. 